\title{
SUBGROUPS OF DIRECT PRODUCTS CLOSELY APPROXIMATED BY DIRECT SUMS
}

\author{
M. FERRER, S. HERNÁNDEZ, AND D. SHAKHMATOV
}

\begin{abstract}
Let $I$ be an infinite set, $\left\{G_{i}: i \in I\right\}$ be a family of (topological) groups and $G=$ $\prod_{i \in I} G_{i}$ be its direct product. For $J \subseteq I, p_{J}: G \rightarrow \prod_{j \in J} G_{j}$ denotes the projection. We say that a subgroup $H$ of $G$ is: (i) uniformly controllable in $G$ provided that for every finite set $J \subseteq I$ there exists a finite set $K \subseteq I$ such that $p_{J}(H)=p_{J}\left(H \cap \bigoplus_{i \in K} G_{i}\right)$; (ii) controllable in $G$ provided that $p_{J}(H)=p_{J}\left(H \cap \bigoplus_{i \in I} G_{i}\right)$ for every finite set $J \subseteq I$; (iii) weakly controllable in $G$ if $H \cap \bigoplus_{i \in I} G_{i}$ is dense in $H$, when $G$ is equipped with the Tychonoff product topology. One easily proves that (i) $\rightarrow$ (ii) $\rightarrow$ (iii). We thoroughly investigate the question as to when these two arrows can be reversed. We prove that the first arrow can be reversed when $H$ is compact, but the second arrow cannot be reversed even when $H$ is compact. Both arrows can be reversed if all groups $G_{i}$ are finite. When $G_{i}=A$ for all $i \in I$, where $A$ is an abelian group, we show that the first arrow can be reversed for all subgroups $H$ of $G$ if and only if $A$ is finitely generated. Connections with coding theory are highlighted.
\end{abstract}

\section{Three Ways to Embed a group into a DiREct product}

Let $\left\{G_{i}: i \in I\right\}$ be a family of groups. As usual, its direct product $G=\prod_{i \in I} G_{i}$ is the set of all functions $g: I \rightarrow \bigcup\left\{G_{i}: i \in I\right\}$ such that $g(i) \in G_{i}$ for every $i \in I$. The group operation on $G$ is defined coordinate-wise: the product $g h \in G$ of $g \in G$ and $h \in G$ is the function defined by $g h(i)=g(i) h(i)$ for each $i \in I$. Clearly, the identity element 1 of $G$ is the function that assigns the identity element of $G_{i}$ to every $i \in I$. The subgroup

$$
\bigoplus_{i \in I} G_{i}=\{g \in G: g(i)=1 \text { for all but finitely many } i \in I\}
$$

of $G$ is called the direct sum of the family $\left\{G_{i}: i \in I\right\}$. For $J \subseteq I$, the projection $p_{I J}: \prod_{i \in I} G_{i} \rightarrow$ $\prod_{j \in J} G_{j}$ defined by $p_{I J}(g)=g\left\lceil J\right.$ for $g \in G$, is the group homomorphism. When $G_{i}=M$ for every $i \in I$, then we write $M^{I}$ instead of $\prod_{i \in I} G_{i}$.

Our first definition introduces two group-theoretic notions that characterize the way a group is embedded into a direct product of groups.

Definition 1.1. Let $I$ be a set, $\left\{G_{i}: i \in I\right\}$ be a family of groups and $G=\prod_{i \in I} G_{i}$ be its direct product. We say that a subgroup $H$ of $G$ is:

(i) controllable in $G$ provided that $p_{I J}(H)=p_{I J}\left(H \cap \bigoplus_{i \in I} G_{i}\right)$ for every finite set $J \subseteq I$;

(ii) uniformly controllable in $G$ provided that for every finite set $J \subseteq I$ there exists a finite set $K \subseteq I$ such that $p_{I J}(H)=p_{I J}\left(H \cap \bigoplus_{i \in K} G_{i}\right)$.

Date: February 4, 2016.

2010 Mathematics Subject Classification. Primary: 22C05; Secondary: 22D35, 54D30, 54D65, 54E35.

Key words and phrases. controllable group, weakly controllable group, finitely generated group, compact group, coding theory.

The first and second named authors acknowledge partial support by the Generalitat Valenciana, grant code: PROMETEO II/2014/062; and by Universitat Jaume I, grant P11B2015-77. The third named author was partially supported by the Grant-in-Aid for Scientific Research (C) No. 22540089 by the Japan Society for the Promotion of Science (JSPS). 
Clearly, a uniformly controllable subgroup is controllable. The following proposition gives two important instances of (uniformly) controllable subgroups of direct products.

Proposition 1.2. Let $\left\{G_{i}: i \in I\right\}$ be a family of groups and $G=\prod_{i \in I} G_{i}$.

(i) Every subgroup of $G$ containing $\bigoplus_{i \in I} G_{i}$ is uniformly controllable in $G$.

(ii) Every subgroup of $\bigoplus_{i \in I} G_{i}$ is controllable in $G$.

Proof. (i) Let $H$ be a subgroup of $G$ such that $\bigoplus_{i \in I} G_{i} \subseteq H$. Let $J \subseteq I$ be a finite set. Then $\bigoplus_{i \in J} G_{i} \subseteq \bigoplus_{i \in I} G_{i} \subseteq H$, and so

$$
\bigoplus_{i \in J} G_{i}=p_{I J}\left(\bigoplus_{i \in I} G_{i}\right) \subseteq p_{I J}(H) \subseteq p_{I J}\left(\prod_{i \in I} G_{i}\right)=\bigoplus_{i \in J} G_{i},
$$

which yields $p_{I J}(H)=\bigoplus_{i \in J} G_{i}=p_{I J}\left(\bigoplus_{i \in J} G_{i}\right)=p_{I J}\left(H \cap \bigoplus_{i \in J} G_{i}\right)$. Therefore, $K=J$ satisfies item (ii) of Definition 1.1.

(ii) If $H$ is a subgroup of $\bigoplus_{i \in I} G_{i}$, then $H=H \cap \bigoplus_{i \in I} G_{i}$, and so item (i) of Definition 1.1 trivially holds.

For future proofs, it would be helpful to restate Definition 1.1 without using the language of projections:

Proposition 1.3. Let $\left\{G_{i}: i \in I\right\}$ be a family of groups and $H$ be a subgroup of its direct product $G=\prod_{i \in I} G_{i}$. Then:

(i) $H$ is controllable in $G$ if and only if for every $h \in H$ and each finite set $J \subseteq I$ there exists $g \in H \cap \bigoplus_{i \in I} G_{i}$ such that $g \uparrow_{J}=h \uparrow_{J}$;

(ii) $H$ is uniformly controllable in $G$ if and only if for every finite set $J \subseteq I$ there exists a finite set $K \subseteq I$ such that for every $h \in H$ one can find $g \in H \cap \bigoplus_{i \in K} G_{i}$ with $g \uparrow_{J}=h \uparrow_{J}$.

When all groups $G_{i}$ in question have some topology, we always equip their direct product $\prod_{i \in I} G_{i}$ with the Tychonoff product topology, and we also consider the following topological property.

Definition 1.4. Let $I$ be a set, $\left\{G_{i}: i \in I\right\}$ be a family of topological groups and $G=\prod_{i \in I} G_{i}$ be its direct product. We say that a subgroup $H$ of $G$ is weakly controllable in $G$ if $H \cap \bigoplus_{i \in I} G_{i}$ is dense in $H$.

The relevance of the topological notion from Definition 1.4 to the group-theoretic notions from Definition 1.1 can be seen from the following proposition which justifies the use of the word "weakly" in Definition 1.4.

Proposition 1.5. A controllable subgroup of an arbitrary direct product $G=\prod_{i \in I} G_{i}$ of topological groups $G_{i}$ is weakly controllable.

Proof. Let $H$ be a controllable subgroup of $G$. We need to show that $H \cap \bigoplus_{i \in I} G_{i}$ is dense in $H$. Let $O$ be an open subset of $G$ with $O \cap H \neq \emptyset$. It suffices to check that $O \cap H \cap \bigoplus_{i \in I} G_{i} \neq \emptyset$. Fix $h \in O \cap H$. By the definition of the product topology, we can find a finite set $J \subseteq I$ and an open neighbourhood $U_{i}$ of $h(i)$ in $G_{i}$ for every $i \in J$ such that

$$
h \in W=\left\{g \in G: g(i) \in U_{i} \text { for all } i \in J\right\} \subseteq O .
$$

Since $H$ is controllable in $G$, Proposition 1.3(i) allows us to find $g \in H \cap \bigoplus_{i \in I} G_{i}$ with $g \uparrow_{J}=h \uparrow_{J}$. From this and (1.1) it follows that $g \in W \subseteq O$. Therefore, $g \in O \cap H \cap \bigoplus_{i \in I} G_{i} \neq \emptyset$.

It is clear from Definition 1.1 that a uniformly controllable subgroup is controllable. Combining this with the last proposition, we obtain the following chain of implications:

$$
\text { uniformly controllable } \rightarrow \text { controllable } \rightarrow \text { weakly controllable. }
$$


These implications show that the three notions introduced above express a degree of how closely a subgroup of a direct product is approximated by its direct sum.

In this paper we study the question whether the two implications above can be reversed for various classes of groups.

The principal result in Section 2 is Theorem 2.6 which asserts that the two notions from Definition 1.1 coincide for compact subgroups of arbitrary direct products $\prod_{i \in I} G_{i}$ of topological groups $G_{i}$. Since both notions from Definition 1.1 are purely algebraic in the sense that they do not depend on topologies of groups $G_{i}$, it is somewhat surprising that a topological property such as compactness imposed on a subgroup of $\prod_{i \in I} G_{i}$ has an influence on the invertibility of the first arrow in (1.2). In particular, this arrow is reversible for closed subgroups of arbitrary products $\prod_{i \in I} G_{i}$ of compact groups $G_{i}$; see Corollary 2.7. When all groups $G_{i}$ are finite, then both arrows in (1.2) can be reversed for arbitrary (not necessarily closed) subgroups of the product $\prod_{i \in I} G_{i}$; see Corollary 2.3. This result has profound applications in coding theory.

The invertibility of the first arrow in (1.2) is thouroughly investigated in Section 3. In Theorem 3.1 we characterize abelian groups $M$ such that every controllable subgroup of an infinite power $M^{I}$ is uniformly controllable; this property holds if and only if $M$ is finitely generated. It follows that, for a non-finitely generated abelian group $M$ and an infinite set $I$, the product $M^{I}$ always contains some subgroup that is controllable but not uniformly controllable. As an application, it follows that the first arrow in (1.2) is not reversible for arbitrary subgroups of the countable power $M^{\mathbb{N}}$ of the compact metric group $M=\mathbb{Z}(2)^{\mathbb{N}}$ of order 2 (Example 3.2), thereby demonstrating that compactness of the subgroup is essential in Theorem 2.6 and closedness of the subgroup is essential in Corollary 2.7. We push this even further in Corollary 3.4 by constructing a large family (having the maximal size $2^{\mathfrak{c}}$ ) of subgroups of the countable power $\mathbb{T}^{\mathbb{N}}$ of the circle group $\mathbb{T}$ each of which is controllable but not uniformly controllable. (Here $\mathfrak{c}$ denotes the cardinality of the continuum.) Both Example 3.2 and Corollary 3.4 demonstrate that finiteness of groups $G_{i}$ in Corollary 2.3 cannot be replaced by their compactness.

In Section 4 we thouroughly investigate the invertibility of the second arrow in (1.2). In Theorem 4.3 we prove that the family $\mathscr{H}$ of all weakly controllable non-controllable subgroups of $\mathbb{T}^{\mathbb{N}}$ has cardinality $2^{\mathfrak{c}}$, which is the maximal size possible. Furthermore, we exhibit a compact member of $\mathscr{H}$ in Example 4.1 and a countable torsion member of $\mathscr{H}$ in Example 4.2.

In Section 5 we study the behaviour of the three notions under taking closures and dense subgroups. The three notions of controllability introduced in this section are closely related to coding theory, and this connection is explained in detail in Section 6. Among other things, this close connection justifies our choice of terminology in Definitions 1.1(i) and 1.4. To the best of our knowledge, the notion of uniform controllability introduced in Definition 1.1(ii) in new and has no analogue in coding theory, although it is weaker then the classical notion of strong controllability; see Definition 6.1(iv) and implications in (6.1). Theorem 7.3 shows that, for every prime number $p$, the family $\mathscr{H}_{p}$ of subgroups of the product $\mathbb{Z}(p)^{\mathbb{N}}$ which are uniformly controllable but not strongly controllable has cardinality $2^{\mathfrak{c}}$, the maximal size possible.

In Section 8 we study the structure of profinite metric abelian groups. We prove that a compact metric profinite abelian group $G$ whose torsion part

$$
t(G)=\{x \in G: n x=0 \text { for some } n \in \mathbb{N} \backslash\{0\}\}
$$

is dense in $G$ is topologically isomorphic to a product of countably many finite cyclic groups; see Theorem 8.2. In particular, a topological abelian group $G$ is topologically isomorphic to a direct product of countably many finite cyclic groups if and only if $G$ is zero-dimensional, compact metric and $t(G)$ is dense in $G$; see Corollary 8.3. As a corollary, we show that a closed weakly controllable subgroup of a countable direct product of finite abelian groups is itself topologically isomorphic to a product of finite (cyclic) abelian groups; see Corollary 9.1. These results should be compared with similar results for the class of Valdivia compact groups obtained recently in $[8,1,9]$. 
Experts in coding theory may want to start with Sections 6 and 9 first, and then proceed with the rest of the paper.

\section{Cases When various forms of CONTROllability COINCIDE}

In this section we investigate special cases when arrows in (1.2) can be reversed.

Recall that a group $G$ satisfies the ascending chain condition provided that every ascending chain $G_{0} \subseteq G_{1} \subseteq \cdots \subseteq G_{n} \subseteq G_{n+1} \subseteq \ldots$ of subgroups of $G$ stabilizes; that is, there exists $k \in \mathbb{N}$ such that $G_{k}=G_{m}$ for all $m \in \mathbb{N}$ with $m \geq k$.

The first arrow in (1.2) can be reversed when all groups $G_{i}$ satisfy the ascending chain condition.

Proposition 2.1. Let $I$ be a set and let $\left\{G_{i}: i \in I\right\}$ be a family of groups such that all $G_{i}$ satisfy the ascending chain condition. Then a subgroup of the direct product $G=\prod_{i \in I} G_{i}$ is controllable in $G$ if and only if it is uniformly controllable in $G$.

Proof. Clearly, every uniformly controllable subgroup of $G$ is controllable in $G$. To prove the reverse implication, assume that $H$ is a controllable subgroup of $G$. Fix a finite subset $J$ of $I$. Since each $G_{i}$ for $i \in J$ satisfies the ascending chain condition, so does the finite product $\prod_{i \in J} G_{i}$. Therefore, the subgroup $p_{I J}(H)$ of $\prod_{i \in J} G_{i}$ is finitely generated. Let $X$ be a finite set of generators for $p_{I J}(H)$. Since $H$ is controllable in $G$, we have $p_{I J}(H)=p_{I J}\left(H \cap \bigoplus_{i \in I} G_{i}\right)$, so we can fix a finite set $Y \subseteq H \cap \bigoplus_{i \in I} G_{i}$ with $X=p_{I J}(Y)$. Since $Y \subseteq \bigoplus_{i \in I} G_{i}$, there exists a finite set $K \subseteq I$ such that $Y \subseteq \bigoplus_{i \in K} G_{i}$. Now

$$
p_{I J}(H)=\langle X\rangle=\left\langle p_{I J}(Y)\right\rangle \subseteq p_{I J}\left(H \cap \bigoplus_{i \in K} G_{i}\right) \subseteq p_{I J}(H),
$$

which yields $p_{I J}(H)=p_{I J}\left(H \cap \bigoplus_{i \in K} G_{i}\right)$. This proves that $H$ is uniformly controllable in $G$.

The second arrow in (1.2) can be reversed when all groups $G_{i}$ are discrete.

Proposition 2.2. Let $I$ be a set and let $\left\{G_{i}: i \in I\right\}$ be a family of discrete groups. Then a subgroup $H$ of the direct product $G=\prod_{i \in I} G_{i}$ is weakly controllable in $G$ if and only if it is controllable in $G$.

Proof. The "if" part is proved in Proposition 1.5. To prove the "only if" part, suppose that $H$ is weakly controllable in $G$. To show that $H$ is controllable in $G$, fix $h \in H$ and a finite set $J \subseteq I$. Since all $G_{i}$ are discrete, it follows from the definition of the product topology that $U=\{g \in G: g(i)=h(i)$ for all $i \in J\}$ is an open subset of $G$. Note that $h \in U \cap H$, so $U \cap H$ is a non-empty open subset of $H$. By the weak controllability of $H$, we can find $g \in U \cap H \cap \bigoplus_{i \in I} G_{i}$. Clearly, $g \uparrow_{J}=h \uparrow_{J}$. Therefore, $H$ is controllable in $G$ by Proposition 1.3(i).

When the groups in question are finite, Proposition 2.2 can be strengthened a bit further, allowing the reversal of both arrows in (1.2).

Corollary 2.3. Let $I$ be a set and let $\left\{G_{i}: i \in I\right\}$ be a family of finite (discrete) groups. Then for every subgroup $H$ of the direct product $G=\prod_{i \in I} G_{i}$ the following conditions are equivalent:

(i) $H$ is weakly controllable;

(ii) $H$ is controllable;

(iii) $H$ is uniformly controllable.

Proof. The equivalence (i) $\leftrightarrow$ (ii) is proved in Proposition 2.2, and the equivalence (ii) $\rightarrow$ (iii) is proved in Proposition 2.1.

The rest of this section is devoted to showing that the first arrow in (1.2) can be reversed for compact subgroups of direct products; see Theorem 2.6. For this end, we shall need a general proposition which has its own interest. 
Proposition 2.4. Let $\left\{G_{i}: i \in I\right\}$ be a family of (topological) groups and let $H$ be a subgroup of its direct product $G=\prod_{i \in I} G_{i}$. Let $S$ be a subset of $I$ and $G_{S}=\prod_{i \in S} G_{i}=p_{I S}(G)$.

(i) If $H$ is (uniformly) controllable in $G$, then $p_{I S}(H)$ is (uniformly) controllable in $G_{S}$.

(ii) If $H$ is weakly controllable in $G$, then $p_{I S}(H)$ is weakly controllable in $G_{S}$.

Proof. (i) For every $K \subseteq I$, one has

$$
p_{I S}\left(H \cap \bigoplus_{i \in K} G_{i}\right) \subseteq p_{I S}(H) \cap \bigoplus_{i \in K \cap S} G_{i}
$$

Let $J \subseteq S$ be a finite set. Since $H$ is (uniformly) controllable, $p_{I J}(H)=p_{I J}\left(H \cap \bigoplus_{i \in K} G_{i}\right)$ holds for some $K \subseteq I$, where $K=I$ when $H$ is controllable and $K$ is finite when $H$ is uniformly controllable. Since $p_{I J}=p_{S J} \circ p_{I S}$, from this and (2.1) we get

$$
p_{S J}\left(p_{I S}(H)\right)=p_{S J}\left(p_{I S}\left(H \cap \bigoplus_{i \in K} G_{i}\right)\right) \subseteq p_{S J}\left(p_{I S}(H) \cap \bigoplus_{i \in K^{\prime}} G_{i}\right) \subseteq p_{S J}\left(p_{I S}(H)\right),
$$

where $K^{\prime}=K \cap S$. This yields $p_{S J}\left(p_{I S}(H)\right)=p_{S J}\left(p_{I S}(H) \cap \bigoplus_{i \in K^{\prime}} G_{i}\right)$. When $K$ is finite, $K^{\prime}$ is also finite, and when $K=I, K^{\prime}=S$. This shows that $p_{I S}(H)$ is (uniformly) controllable in $G_{S}$.

In the proof of (ii) one uses the fact that the projection $p_{I S}: G \rightarrow G_{S}$ is continuous, and so it preserves density.

We need to prove the special case of Theorem 2.6 first.

Lemma 2.5. Let $I$ be a countable set and $\left\{G_{i}: i \in I\right\}$ be a family of topological groups. Then every compact controllable subgroup of $G=\prod_{i \in I} G_{i}$ is uniformly controllable.

Proof. Let $H$ be a compact controllable subgroup of $G$. Fix a finite subset $J$ of $I$. We need to find a finite set $K \subseteq I$ such that $p_{I J}(H)=p_{I J}\left(H \cap \bigoplus_{i \in K} G_{i}\right)$.

For every finite subset $F$ of $I, H_{F}=H \cap \bigoplus_{i \in F} G_{i}$ is a closed (and thus, compact) subgroup of $H$, so its continuous homomorphic image $p_{I J}\left(H_{F}\right)$ is a compact (and thus, closed) subgroup of $p_{I J}(H)$. The latter group is compact as well, as a continuous image of the compact group $H$. Note that $H \cap \bigoplus_{i \in I} G_{i}=\bigcup\left\{H_{F}: F \subseteq I\right.$ is finite $\}$. Since $H$ is controllable,

$$
p_{I J}(H)=p_{I J}\left(H \cap \bigoplus_{i \in I} G_{i}\right)=\bigcup\left\{p_{I J}\left(H_{F}\right): F \subseteq I \text { is finite }\right\} .
$$

Since the collection of finite subsets of the countable set $I$ is countable, applying (2.2) and the Baire category theorem to $p_{I J}(H)$, we can find a finite set $F^{*} \subseteq I$ such that $N=p_{I J}\left(H_{F^{*}}\right)$ has non-empty interior in $p_{I J}(H)$. Since $N$ is a subgroup of $p_{I J}(H)$, it must be open in $p_{I J}(H)$. Since the latter group is compact, $N$ has finite index in $p_{I J}(H)$; that is, there exists a finite set $Y \subseteq p_{I J}(H)$ such that $p_{I J}(H)=Y N$. Applying (2.2) we can find a finite set $X \subseteq H \cap \bigoplus_{i \in I} G_{i}$ with $Y=p_{I J}(X)$. Finally, let $K$ be a finite subset of $I$ such that $F^{*} \subseteq K$ and $X \subseteq \bigoplus_{i \in K} G_{i}$.

Let $h \in H$ be arbitrary. Since $p_{I J}(h) \in p_{I J}(H)=Y N$, there exists $y \in Y$ and $z \in N$ with $p_{I J}(h)=y z$. Pick $x \in X \subseteq H \cap \bigoplus_{i \in K} G_{i}$ and $h^{*} \in H_{F^{*}} \subseteq H \cap \bigoplus_{i \in K} G_{i}$ such that $y=p_{I J}(x)$ and $z=p_{I J}\left(h^{*}\right)$. Since $H$ is a subgroup of $G$, so is $H \cap \bigoplus_{i \in K} G_{i}$. Hence, $h^{\prime}=x h^{*} \in H \cap \bigoplus_{i \in K} G_{i}$. Finally note that $p_{I J}\left(h^{\prime}\right)=p_{I J}\left(x h^{*}\right)=p_{I J}(x) p_{I J}\left(h^{*}\right)=y z=p_{I J}(h)$. This shows that $p_{I J}(H) \subseteq$ $p_{I J}\left(H \cap \bigoplus_{i \in K} G_{i}\right)$. The reverse inclusion is obvious.

Theorem 2.6. Let $\left\{G_{i}: i \in I\right\}$ be a family of topological groups and $G=\prod_{i \in I} G_{i}$ be its direct product. For a compact subgroup $H$ of $G$, the following conditions are equivalent:

(i) $H$ is controllable in $G$;

(ii) $H$ is uniformly controllable in $G$. 
Proof. The implication (ii) $\rightarrow$ (i) is trivial. To check the implication (i) $\rightarrow$ (ii), we assume that $H$ is not uniformly controllable in $G$, and we shall prove that $H$ is not controllable in $G$ either.

Since $H$ is not uniformly controllable in $G$, we can fix a finite set $J \subseteq I$ such that

$$
p_{I J}(H) \neq p_{I J}\left(H \cap \bigoplus_{i \in K} G_{i}\right) \text { for every finite set } K \subseteq I .
$$

For each $F \subseteq I$ define

$$
N_{F}=\{h \in H: h(i)=1 \text { for all } i \in F\}
$$

and note that $N_{F}$ is a closed subgroup of $H$.

Claim 1. For every finite set $K \subseteq I$ there exists a finite set $F \subseteq I$ disjoint from $K$ such that $p_{I J}(H) \backslash p_{I J}\left(N_{F}\right) \neq \emptyset$.

Proof. Use (2.3) to fix $h_{K} \in H$ such that $p_{I J}\left(h_{K}\right) \notin p_{I J}\left(H \cap \bigoplus_{i \in K} G_{i}\right)$. Then

$$
\Phi_{K}=\left\{h \in H: p_{I J}(h)=p_{I J}\left(h_{K}\right)\right\}=H \cap p_{I J}^{-1}\left(p_{I J}\left(h_{K}\right)\right)
$$

is a closed subset of $H$ such that $\Phi_{K} \cap \bigoplus_{i \in K} G_{i}=\emptyset$. Since $H$ is compact, so is $\Phi_{K}$.

Observe that $\mathscr{N}_{K}=\left\{N_{S}: S \subseteq I \backslash K\right.$ is finite $\}$ is a family of closed subsets of $H$ having the finite intersection property. Clearly, $\bigcap \mathscr{N}_{K} \subseteq \bigoplus_{i \in K} G_{i}$, which yields $\Phi_{K} \cap \cap \mathscr{N}_{K}=\emptyset$. Since $\Phi_{K}$ is compact, $\Phi_{K} \cap N_{F}=\emptyset$ for some finite set $F \subseteq I \backslash K$.

Let $h \in N_{F}$ be arbitrary. Then $h \notin \Phi_{K}$, and since $h \in H,(2.5)$ implies that $p_{I J}(h) \neq p_{I J}\left(h_{K}\right)$. This shows that $p_{I J}\left(h_{K}\right) \notin p_{I J}\left(N_{F}\right)$. Since $p_{I J}\left(h_{K}\right) \in p_{I J}(H)$, we get $p_{I J}\left(h_{K}\right) \in p_{I J}(H) \backslash$ $p_{I J}\left(N_{F}\right) \neq \emptyset$.

Let $[I]^{<\omega}$ be the family of all finite subsets of $I$. Our claim allows us to define a map $\sigma:[I]^{<\omega} \rightarrow$ $[I]^{<\omega}$ such that

$$
K \cap \sigma(K)=\emptyset \text { and } p_{I J}(H) \backslash p_{I J}\left(N_{\sigma(K)}\right) \neq \emptyset \text { for all } K \in[I]^{<\omega} .
$$

Let $S_{0}=J$ and let $S_{n+1}=S_{n} \cup \sigma\left(S_{n}\right)$ for every $n \in \mathbb{N}$. The set $S=\bigcup\left\{S_{n}: n \in \mathbb{N}\right\}$ is at most countable. Clearly, $J=S_{0} \subseteq S$.

Claim 2. The subgroup $H_{S}=p_{I S}(H)$ of $G_{S}=p_{I S}(G)=\prod_{i \in S} G_{i}$ is not uniformly controllable in $G_{S}$.

Proof. Since $J$ is a finite subset of $S$, it suffices to show that

$$
p_{S J}\left(H_{S}\right) \backslash p_{S J}\left(H_{S} \cap \bigoplus_{i \in K} G_{i}\right) \neq \emptyset
$$

for every finite set $K \subseteq S$. Fix such a $K$. Since the sequence $\left\{S_{n}: n \in \mathbb{N}\right\}$ is monotonically increasing and $S=\bigcup\left\{S_{n}: n \in \mathbb{N}\right\}$, there exists $n \in \mathbb{N}$ with $K \subseteq S_{n}$.

We claim that

$$
p_{S J}\left(H_{S} \cap \bigoplus_{i \in K} G_{i}\right) \subseteq p_{I J}\left(N_{\sigma\left(S_{n}\right)}\right) .
$$

Indeed, let $g \in H_{S} \cap \bigoplus_{i \in K} G_{i}$ be arbitrary. Since $g \in H_{S}=p_{I S}(H)$, we can choose $h \in H$ such that $g=p_{I S}(h)$; that is, $h\left\lceil_{S}=g\right.$. Since $K \subseteq S_{n}, \sigma\left(S_{n}\right) \cap S_{n}=\emptyset$ and $g \in \bigoplus_{i \in K} G_{i}$, we conclude that $g(i)=1$ for all $i \in \sigma\left(S_{n}\right)$. Since $\sigma\left(S_{n}\right) \subseteq S_{n+1} \subseteq S$, it follows that $h(i)=g(i)=1$ for all $i \in \sigma\left(S_{n}\right)$. Hence, $h \in N_{\sigma\left(S_{n}\right)}$ by $(2.4)$. Therefore, $p_{S J}(g)=p_{S J}\left(p_{I S}(h)\right)=p_{I J}(h) \in p_{I J}\left(N_{\sigma\left(S_{n}\right)}\right)$. This finishes the proof of (2.8). 
From (2.6) and (2.8), we obtain

$$
\emptyset \neq p_{I J}(H) \backslash p_{I J}\left(N_{\sigma\left(S_{n}\right)}\right) \subseteq p_{I J}(H) \backslash p_{S J}\left(H_{S} \cap \bigoplus_{i \in K} G_{i}\right) .
$$

Since $p_{I J}(H)=p_{S J}\left(p_{I S}(H)\right)=p_{S J}\left(H_{S}\right)$, this gives $(2.7)$.

As a continuous image of the compact group $H$, the subgroup $H_{S}$ of $G_{S}=\prod_{i \in S} G_{i}$ is compact. Since $S$ is countable, it follows from Lemma 2.5 and Claim 2 that $H_{S}$ is not controllable in $G_{S}$. Combining this with Proposition 2.4(i), we obtain that $H$ is not controllable in $G$.

Corollary 2.7. Let $\left\{G_{i}: i \in I\right\}$ be a family of compact groups. Then a closed subgroup $H$ of $G=\prod_{i \in I} G_{i}$ is controllable in $G$ if and only if $H$ is uniformly controllable in $G$.

\section{Controllability vs uniform controllability}

In this section we characterize abelian groups $M$ such that every controllable subgroup of the power $M^{I}$ is uniformly controllable; see Theorem 3.1. First, we develop some machinery necessary for the proof of this theorem.

Let $M$ be an abelian group, and let $\mathscr{A}=\left\{A_{i}: i \in \mathbb{N}\right\}$ be a strictly ascending chain of subgroups of $M$; that is,

$$
A_{0} \subsetneq A_{1} \subsetneq A_{2} \subsetneq \cdots \subsetneq A_{i} \subsetneq A_{i+1} \subsetneq \ldots
$$

and each $A_{i}$ is a subgroup of $M$. For each $i \in \mathbb{N}$,

$$
H_{\mathscr{A}, i}=\left\{(a, a, \ldots, a) \in M^{i+1}: a \in A_{i}\right\} \times \prod_{j=i+1}^{\infty}\{0\}
$$

is a subgroup of $G=M^{\mathbb{N}}$. Clearly,

$$
H_{\mathscr{A}}=\left\langle\bigcup_{i \in \mathbb{N}} H_{\mathscr{A}, i}\right\rangle
$$

is a subgroup of $G$ associated with the chain $\mathscr{A}$. Note that every element $h \in H_{\mathscr{A}}$ has a representation

$$
h=\sum_{i=0}^{m} h_{i}, \text { where } h_{i} \in H_{\mathscr{A}, i} \text { for all } i=0, \ldots, m \text { and } h_{m} \neq 0 .
$$

We claim that

$$
H_{\mathscr{A}} \cap \bigoplus_{i=0}^{k} M=\sum_{i=0}^{k} H_{\mathscr{A}, i} \quad \text { for all } k \in \mathbb{N} .
$$

Fix $k \in \mathbb{N}$. Let $h$ be an element of the set on the left hand side of (3.5). Then $h$ has a representation as in (3.4). Note that $h_{m} \in H_{\mathscr{A}, m},(3.2)$ and $h_{m} \neq 0$ yield $h_{m}(m) \neq 0$. Now (3.2) and (3.4) give $h(m)=\sum_{i=0}^{m} h_{i}(m)=h_{m}(m) \neq 0$. Since $h \in \bigoplus_{i=0}^{k} M$, from this we conclude that $m \leq k$. Combining this with (3.4), we obtain $h \in \sum_{i=0}^{m} H_{\mathscr{A}, i} \subseteq \sum_{i=0}^{k} H_{\mathscr{A}, i}$. This argument proves that the set on the left hand side of (3.5) is a subset of the set on the right hand side of the same equation. The converse inclusion follows easily from (3.2) and (3.3).

Claim $3 . \quad$ (i) $H_{\mathscr{A}}$ is controllable in $G$ but not uniformly controllable in $G$.

(ii) If all $A_{i}(i \in \mathbb{N})$ are countable, then $H_{\mathscr{A}}$ is countable as well. 
Proof. (i) Since $H_{\mathscr{A}} \subseteq \bigoplus_{k \in \mathbb{N}} M$, the subgroup $H_{\mathscr{A}}$ of $G$ is controllable in $G$ by Proposition 1.2(ii). Let $J=\{0\}$. Let $K$ be an arbitrary finite subset of $\mathbb{N}$. Then $K \subseteq\{0,1, \ldots, k\}$ for some $k \in \mathbb{N}$. Combining this with (3.1), (3.2), (3.5) and recalling the fact that $p_{\mathbb{N} J}$ is a homomorphism, we get

$$
p_{\mathbb{N} J}\left(H_{\mathscr{A}} \cap \bigoplus_{i \in K} M\right) \subseteq p_{\mathbb{N} J}\left(H_{\mathscr{A}} \cap \bigoplus_{i=0}^{k} M\right)=p_{\mathbb{N} J}\left(\sum_{i=0}^{k} H_{\mathscr{A}, i}\right)=\sum_{i=0}^{k} p_{\mathbb{N} J}\left(H_{\mathscr{A}, i}\right)=\sum_{i=0}^{k} A_{i}=A_{k},
$$

where the last equation follows from the fact that $A_{k}$ is a subgroup of $M$. Similarly, since all $A_{i}$ $(i \in \mathbb{N})$ are subgroups of $M$ and $p_{\mathbb{N} J}$ is a homomorphism, from (3.1), (3.2) and (3.3), we obtain

$$
p_{\mathbb{N} J}\left(H_{\mathscr{A}}\right)=\left\langle\bigcup_{i \in \mathbb{N}} p_{\mathbb{N} J}\left(H_{\mathscr{A}, i}\right)\right\rangle=\left\langle\bigcup_{i \in \mathbb{N}} A_{i}\right\rangle=\bigcup_{i \in \mathbb{N}} A_{i} .
$$

Since $A_{k}$ is a proper subset of $\bigcup_{i \in \mathbb{N}} A_{i}$ by (3.1), we deduce that

$$
p_{\mathbb{N} J}\left(H_{\mathscr{A}}\right) \neq p_{\mathbb{N} J}\left(H_{\mathscr{A}} \cap \bigoplus_{i \in K} M\right) \text {. }
$$

This shows that $H_{\mathscr{A}}$ is not uniformly controllable in $G$.

(ii) This easily follows from (3.2) and (3.3).

Theorem 3.1. For an abelian group $M$, a set $I$ and the group $G=M^{I}$, the following conditions are equivalent:

(i) every countable controllable subgroup of $G$ is uniformly controllable in $G$;

(ii) every controllable subgroup of $G$ is uniformly controllable in $G$;

(iii) either $I$ is finite or $M$ is finitely generated.

Proof. (i) $\rightarrow$ (iii) We assume that (iii) fails, and we shall prove that (i) fails as well. Since (iii) fails, the set $I$ is infinite and the group $M$ is not finitely generated. The latter assumption allows us to find a chain $\mathscr{A}=\left\{A_{k}: k \in \mathbb{N}\right\}$ of finitely generated subgroups $A_{k}$ of $M$ satisfying (3.1). By Claim 3 , the corresponding subgroup $H_{\mathscr{A}}$ of $M^{\mathbb{N}}$ is controllable in $M^{\mathbb{N}}$ but not uniformly controllable in $M^{\mathbb{N}}$. Since $I$ is infinite, we can fix a countably infinite subset $N$ of $I$. Let $\varphi: N \rightarrow \mathbb{N}$ be a bijection. It naturally induces the group isomorphism $\theta: M^{\mathbb{N}} \rightarrow M^{N}$ defined by $\theta(h)=h \circ \varphi$ for $h \in M^{\mathbb{N}}$. One easily checks that $H^{\prime}=\theta\left(H_{\mathscr{A}}\right)$ is a controllable subgroup of $M^{N}$ that is not uniformly controllable in $M^{N}$. Now $H=H^{\prime} \times\{\mathbf{0}\}$ is a controllable subgroup of $G$ that is not uniformly controllable in $G$. (Here $\mathbf{0}$ is the zero element of the group $M^{I \backslash N}$.) Therefore, (i) fails.

(iii) $\rightarrow$ (ii) If the set $I$ is finite, then (ii) trivially holds, as one can take $K=I$ in the definition of uniform controllability. If the group $M$ is finitely generated, then it satisfies the ascending chain condition. Applying Proposition 2.1, we conclude that (ii) holds.

(ii) $\rightarrow$ (i) is trivial.

The next example demonstrates that compactness of $H$ is essential in Theorem 2.6 and closedness of $H$ is essential in Corollary 2.7.

Example 3.2. Let $M=\mathbb{Z}(2)^{\mathbb{N}}$ be the infinite compact metric group of order 2. Since $M$ is not finitely generated, Theorem 3.1 implies that $G=M^{\mathbb{N}}$ has a countable subgroup $H$ which is controllable in $G$ but not uniformly controllable in $G$. Clearly, $H$ has order 2 as well.

Item (i) of our next theorem demonstrates that whenever the countable power $G=M^{\mathbb{N}}$ of some abelian group $M$ has at least one subgroup that is controllable in $G$ but is not uniformly controllable in $G$, then the family of all such subgroups is rather large. Item (ii) of this theorem establishes the version of this fact for countable subgroups of $G$.

Theorem 3.3. Let $M$ be an abelian group, $\mathscr{H}$ be the family of all subgroups of $G=M^{\mathbb{N}}$ which are controllable in $G$ but not uniformly controllable in $G$, and let $\mathscr{H}_{c}=\{H \in \mathscr{H}:|H| \leq|\mathbb{N}|\}$. 
(i) Either $\mathscr{H}=\emptyset$ or $|\mathscr{H}| \geq 2^{|M|} \geq \mathfrak{c}$.

(ii) If $M$ is countably infinite, then either $\mathscr{H}_{c}=\emptyset$ or $\left|\mathscr{H}_{c}\right|=\mathfrak{c}$.

Proof. Suppose that $\mathscr{H} \neq \emptyset$. (In particular, this assumption trivially holds when $\mathscr{H}_{c} \neq \emptyset$.) Applying Theorem 3.1, we conclude that $M$ is not finitely generated. In particular, $M$ is infinite, and so $2^{|M|} \geq \mathfrak{c}$. We consider two cases, depending on the cardinality of the group $M$.

Case 1. $M$ is countably infinite. In this case, $2^{|M|}=\mathfrak{c}$. To prove both items (i) and (ii), it suffices to show that $\left|\mathscr{H}_{c}\right| \geq \mathfrak{c}$. Indeed, $|\mathscr{H}| \geq\left|\mathscr{H}_{c}\right|$, which yields (i). Since $\left|M^{\mathbb{N}}\right|=|\mathbb{N}|^{|\mathbb{N}|}=\mathfrak{c}$, we have $\left|\mathscr{H}_{c}\right| \leq\left|M^{\mathbb{N}}\right|^{\omega}=\mathfrak{c}^{\omega}=\mathfrak{c}$. Combining it with $\left|\mathscr{H}_{c}\right| \geq \mathfrak{c}$, we get (ii).

Since $M$ is not finitely generated, by Theorem 3.1 the product $M^{\mathbb{N}}$ contains a countable subgroup $K$ that is controllable in $M^{\mathbb{N}}$ but not uniformly controllable in $M^{\mathbb{N}}$. Now we proceed similarly to the proof of the implication (i) $\rightarrow$ (iii) of Theorem 3.1. The family $\mathscr{N}$ of all infinite subsets of $\mathbb{N}$ has cardinality c. For every $N \in \mathscr{N}$ we fix a bijection $\varphi_{N}: N \rightarrow \mathbb{N}$, which induces the group isomorphism $\theta_{N}: M^{\mathbb{N}} \rightarrow M^{N}$ defined by $\theta_{N}(h)=h \circ \varphi_{N}$ for $h \in M^{\mathbb{N}}$. One easily checks that $H_{N}^{\prime}=\theta_{N}(K)$ is a countable controllable subgroup of $M^{N}$ that is not uniformly controllable in $M^{N}$. Now $H_{N}=H_{N}^{\prime} \times\left\{\mathbf{0}_{N}\right\}$ is a countable controllable subgroup of $G$ that is not uniformly controllable in $G$. (Here $\mathbf{0}_{N}$ is the zero element of the group $M^{\mathbb{N} \backslash N}$.) Therefore, $\left\{H_{N}: N \in \mathscr{N}\right\} \subseteq \mathscr{H}_{c}$. Finally, one easily sees that $H_{N_{1}} \neq H_{N_{2}}$ whenever $N_{1}, N_{2} \in \mathscr{N}$ and $N_{1} \neq N_{2}$. This proves that $\left|\mathscr{H}_{c}\right| \geq\left|\left\{H_{N}: N \in \mathscr{N}\right\}\right|=|\mathscr{N}|=\mathfrak{c}$.

Case 2. $M$ is uncountable. In this case, $|M|=\sup \left\{r_{p}(M): p \in \mathbb{P} \cup\{0\}\right\}$, where $\mathbb{P}$ is the set of all prime numbers and $r_{p}(M)$ is a $p$-rank of $M$. For every $p \in \mathbb{P} \cup\{0\}$ choose a $p$-free subset $Y_{p}$ of $M$ with $\left|Y_{p}\right|=r_{p}(M)$. Define $Y=\bigcup\left\{Y_{p}: p \in \mathbb{P} \cup\{0\}\right\}$. Then $|Y|=\sup \left\{r_{p}(M): p \in \mathbb{P} \cup\{0\}\right\}=|M|$ and the set $Y$ is independent; that is, $\langle Z\rangle \cap\langle Y \backslash Z\rangle=\{0\}$ for every non-empty set $Z \subseteq Y$.

Define $\mathscr{Z}=\{Z \subseteq Y: Z$ is infinite $\}$. Let $Z \in \mathscr{Z}$. Since $Z$ is infinite, we can choose a strictly increasing sequence $\left\{Z_{i}: i \in \mathbb{N}\right\}$ of subsets of $Z$ such that $Z=\bigcup_{i \in \mathbb{N}} Z_{i}$. Since $Z \subseteq Y$ and $Y$ is independent, $\mathscr{A}(Z)=\left\{\left\langle Z_{i}\right\rangle: i \in \mathbb{N}\right\}$ is a strictly ascending chain of subgroups of $M$ with $\bigcup \mathscr{A}(Z)=\langle Z\rangle$. By Claim 3, $H_{\mathscr{A}(Z)} \in \mathscr{H}$. Therefore, $|\mathscr{H}| \geq\left|\left\{H_{\mathscr{A}(Z)}: Z \in \mathscr{Z}\right\}\right|$. Since $|\mathscr{Z}|=2^{|Y|}=2^{|M|}$, it remains only check that $H_{\mathscr{A}\left(Z_{0}\right)} \neq H_{\mathscr{A}\left(Z_{1}\right)}$ whenever $Z_{0}, Z_{1} \in \mathscr{Z}$ and $Z_{0} \neq Z_{1}$. Indeed, there exists $i=0,1$ such that $Z_{i} \backslash Z_{1-i} \neq \emptyset$. Since $Z_{0} \cup Z_{1} \subseteq Y$ and $Y$ is independent, it follows that $Z_{i} \backslash\left\langle Z_{1-i}\right\rangle \neq \emptyset$. Let $J=\{0\}$. Since $p_{\mathbb{N} J}\left(H_{\mathscr{A}\left(Z_{j}\right)}\right)=\left\langle Z_{j}\right\rangle$ for $j=0,1$, we conclude that $p_{\mathbb{N} J}\left(H_{\mathscr{A}\left(Z_{i}\right)}\right) \backslash p_{\mathbb{N} J}\left(H_{\mathscr{A}\left(Z_{1-i}\right)}\right) \neq \emptyset$. This implies that $H_{\mathscr{A}\left(Z_{0}\right)} \neq H_{\mathscr{A}\left(Z_{1}\right)}$.

Corollary 3.4. Let $\mathscr{H}$ be the family of all subgroups of $\mathbb{T}^{\mathbb{N}}$ that are controllable in $\mathbb{T}^{\mathbb{N}}$ but not uniformly controllable in $\mathbb{T}^{\mathbb{N}}$. Then $|\mathscr{H}|=2^{\mathfrak{c}}$.

Proof. Since $\mathbb{T}$ is not finitely generated, $\mathscr{H} \neq \emptyset$ by Theorem 3.1. Therefore, $|\mathscr{H}| \geq 2^{|\mathbb{T}|}=2^{\mathfrak{c}}$ by Theorem 3.3. Since $\left|\mathbb{T}^{\mathbb{N}}\right|=\mathfrak{c}$, the inverse inclusion $|\mathscr{H}| \leq 2^{\left|\mathbb{T}^{\mathbb{N}}\right|}=2^{\mathfrak{c}}$ also holds.

\section{WEAK CONTROLLABILITy VS CONTROLLABILITY}

In this section we demonstrate that the notions or weak controllability and controllability differ for subgroups of $\mathbb{T}^{\mathbb{N}}$, thereby showing that the assumption that each $G_{i}$ is discrete in Proposition 2.2 cannot be replaced with the assumption that each $G_{i}$ is compact, and the assumption that each $G_{i}$ is finite in Corollary 2.3 cannot be weakened to the assumption that each $G_{i}$ is compact, even in the abelian case. In fact, we prove that the family $\mathscr{H}$ of all weakly controllable non-controllable subgroups of $\mathbb{T}^{\mathbb{N}}$ has cardinality $2^{\mathfrak{c}}$, which is the maximal size possible. Furthermore, we exhibit a compact member of $\mathscr{H}$ in Example 4.1 and a countable torsion member of $\mathscr{H}$ in Example 4.2.

First, we develop some machinery necessary for proving these results. 
Let $Y=\left\{y_{k}: k \in \mathbb{N}\right\} \subseteq t(\mathbb{T}) \backslash\{0\}$ be a sequence converging to 0 in $\mathbb{T}$. For every $k \in \mathbb{N}$ define $f_{k} \in \mathbb{T}^{\mathbb{N}}$ by letting

$$
f_{k}(n)=\left\{\begin{array}{ll}
y_{k} & \text { if } n \leq k \\
0, & \text { if } n>k
\end{array} \quad \text { for } n \in \mathbb{N}\right.
$$

Since $Y \subseteq t(\mathbb{T})$, for every $k \in \mathbb{N}$ we can fix $s_{k} \in \mathbb{N}$ such that $s_{k} y_{k}=0$. From this and (4.1), it follows that $s_{k} f_{k}=0$ for every $k \in \mathbb{N}$. Therefore,

$$
\left\langle f_{k}\right\rangle=\left\{m f_{k}: m \in \mathbb{N}, 0 \leq m<s_{k}\right\} \text { for every } k \in \mathbb{N} .
$$

In particular,

$$
F_{Y}=\left\{f_{k}: k \in \mathbb{N}\right\} \subseteq t\left(\mathbb{T}^{\mathbb{N}}\right)
$$

Let $D_{Y}=\left\langle F_{Y}\right\rangle$.

Claim 4. If $g \in \overline{D_{Y}}$, then $g(n)-g(n+1) \in\left\langle y_{n}\right\rangle$ for every $n \in \mathbb{N}$.

Proof. Let $g \in \overline{D_{Y}}$ and $n \in \mathbb{N}$. Since $g \in \overline{D_{Y}} \subseteq \mathbb{T}^{\mathbb{N}}$ and $\mathbb{T}^{\mathbb{N}}$ is metric, we can fix a sequence $\left\{h_{j}: j \in \mathbb{N}\right\} \subseteq D_{Y}$ converging to $g$. For each $j \in \mathbb{N}$, from $h_{j} \in D_{Y}=\left\langle F_{Y}\right\rangle=\left\langle\left\{f_{k}: k \in \mathbb{N}\right\}\right\rangle$ we conclude that there exists a representation $h_{j}=\sum_{k \in \mathbb{N}} m_{j, k} f_{k}$ such that $\left\{m_{j, k}: k \in \mathbb{N}\right\} \subseteq \mathbb{N}$ and the set $\left\{k \in \mathbb{N}: m_{j, k} \neq 0\right\}$ is finite; moreover, by (4.2), we may assume, without loss of generality, that $0 \leq m_{j, k}<s_{k}$ for all $k \in \mathbb{N}$. Since $m_{j, n} \in\left\{0,1, \ldots, s_{n}-1\right\}$ for all $j \in \mathbb{N}$, there exist $m \in \mathbb{N}$ and an infinite set $J \subseteq \mathbb{N}$ such that $m_{j, n}=m$ for all $j \in J$. Since the sequence $\left\{h_{j}: j \in \mathbb{N}\right\}$ converges to $g$, its subsequence $\left\{h_{j}: j \in J\right\}$ converges to $g$ as well.

Let $j \in J$ be arbitrary. By (4.1),

$$
h_{j}(n)=\sum_{k \in \mathbb{N}} m_{j, k} f_{k}(n)=\sum_{k \leq n-1} m_{j, k} f_{k}(n)+m_{j, n} f_{n}(n)+\sum_{k \geq n+1} m_{j, k} f_{k}(n)=m y_{n}+\sum_{k \geq n+1} m_{j, k} y_{k}
$$

and

$$
h_{j}(n+1)=\sum_{k \in \mathbb{N}} m_{j, k} f_{k}(n+1)=\sum_{k \leq n} m_{j, k} f_{k}(n+1)+\sum_{k \geq n+1} m_{j, k} f_{k}(n+1)=\sum_{k \geq n+1} m_{j, k} y_{k},
$$

so $h_{j}(n)-h_{j}(n+1)=m y_{n} \in\left\langle y_{n}\right\rangle$. Since $\left\langle y_{n}\right\rangle$ is a finite subgroup of $\mathbb{T}$, it is closed in $\mathbb{T}$, and so

$$
g(n)-g(n+1)=\lim _{j \rightarrow \infty, j \in J} h_{j}(n)-\lim _{j \rightarrow \infty, j \in J} h_{j}(n+1)=\lim _{j \rightarrow \infty, j \in J}\left(h_{j}(n)-h_{j}(n+1)\right) \in\left\langle y_{n}\right\rangle,
$$

as required.

For every $x \in \mathbb{T}$, define $c_{x} \in \mathbb{T}^{\mathbb{N}}$ by letting $c_{x}(n)=x$ for all $n \in \mathbb{N}$.

Claim 5. Suppose that $x \in \mathbb{T} \backslash\langle Y\rangle$ and $H$ is a subgroup of $\mathbb{T}^{\mathbb{N}}$ such that $D_{Y} \subseteq H \subseteq \overline{D_{Y}}$ and $c_{x} \in H$. Then $H$ is weakly controllable but not controllable.

Proof. Note that $F_{Y} \subseteq \bigoplus_{n \in \mathbb{N}} \mathbb{T}$ by (4.1) and (4.3), so $D_{Y}=\left\langle F_{Y}\right\rangle \subseteq \bigoplus_{n \in \mathbb{N}} \mathbb{T}$ as well. Since $D_{Y} \subseteq H$, we get $D_{Y} \subseteq H \cap \bigoplus_{n \in \mathbb{N}} \mathbb{T}$. Since $D_{Y}$ is dense in $H$, we conclude that $H \cap \bigoplus_{n \in \mathbb{N}} \mathbb{T}$ is dense in $H$ as well. This shows that $H$ is weakly controllable.

Suppose that $H$ is controllable. Applying the definition of controllability to $J=\{0\}$, we get $x=p_{\mathbb{N} J}\left(c_{x}\right) \in p_{\mathbb{N} J}(H)=p_{\mathbb{N} J}\left(H \cap \bigoplus_{i \in \mathbb{N}} \mathbb{T}\right)$, so there exists $g \in H \cap \bigoplus_{i \in \mathbb{N}} \mathbb{T}$ such that $x=p_{\mathbb{N} J}(g)=$ $g(0)$. Since $g \in \bigoplus_{i \in \mathbb{N}} \mathbb{T}$, there exists $n \in \mathbb{N}$ such that $g(i)=0$ for all integers $i>n$. In particular,

$$
x=g(0)=g(0)-g(n+1) .
$$

Since $g \in H \subseteq \overline{D_{Y}}$, from Claim 4 we conclude that $g(i)-g(i+1) \in\left\langle y_{i}\right\rangle$ for all $i=0, \ldots, n$. Therefore,

$$
g(0)-g(n+1)=\sum_{i=0}^{n}(g(i)-g(i+1))=\left\langle y_{0}\right\rangle+\left\langle y_{1}\right\rangle+\cdots+\left\langle y_{n}\right\rangle \subseteq\langle Y\rangle
$$


Combining this with (4.4), we get $x \in\langle Y\rangle$, in contradiction with our assumption on $x$. This contradiction shows that $H$ is not controllable.

Claim 6. $c_{x} \in \overline{D_{Y}}$ for all $x \in \mathbb{T}$.

Proof. Fix $x \in \mathbb{T}$. Let $O$ be an open neighbourhood of $c_{x}$ in $\mathbb{T}^{\mathbb{N}}$. There exist $n \in \mathbb{N}$ and an open neighbourhood $U$ of $x$ in $\mathbb{T}$ such that

$$
V=\left\{f \in \mathbb{T}^{\mathbb{N}}: f(i) \in U \text { for all } i=0, \ldots, n\right\} \subseteq O .
$$

Since $\lim _{k \rightarrow \infty} y_{k}=0$, we can choose $k \in \mathbb{N}$ such that $k \geq n$ and $\left\langle y_{k}\right\rangle \cap U \neq \emptyset$. Fix $m \in \mathbb{Z}$ such that $m y_{k} \in U$. By (4.1), $m f_{k}(i)=m y_{k} \in U$ for every $i=0, \ldots, n$. Therefore, $m f_{k} \in V \subseteq O$ by (4.5). Since $m f_{k} \in D_{Y}$, we have $O \cap D_{Y} \neq \emptyset$. This shows that $c_{x} \in \overline{D_{Y}}$.

Claim 7. If $X \subseteq \mathbb{T}$ and $X \backslash\langle Y\rangle \neq \emptyset$, then $H=\left\langle F_{Y} \cup\left\{c_{x}: x \in X\right\}\right\rangle$ is a weakly controllable subgroup of $\mathbb{T}^{\mathbb{N}}$ that is not controllable.

Proof. Clearly, $D_{Y}=\left\langle F_{Y}\right\rangle \subseteq H$. Let us check the inclusion $H \subseteq \overline{D_{Y}}$. Obviously, $F_{Y} \subseteq D_{Y} \subseteq \overline{D_{Y}}$. Furthermore, $\left\{c_{x}: x \in X\right\} \subseteq \overline{D_{Y}}$ by Claim 6. Since $D_{Y}$ is a subgroup of $\mathbb{T}^{\mathbb{N}}$, so is its closure $\overline{D_{Y}}$. Therefore, $H=\left\langle F_{Y} \cup\left\{c_{x}: x \in X\right\}\right\rangle \subseteq \overline{D_{Y}}$. Since $X \backslash\langle Y\rangle \neq \emptyset$, there exists $x \in X \backslash\langle Y\rangle$. Since $c_{x} \in H$, Claim 5 can be applied.

Example 4.1. Let $Y=\left\{y_{k}: k \in \mathbb{N}\right\} \subseteq t(\mathbb{T})$ be any sequence converging to 0 in $\mathbb{T}$. Then $H=\overline{D_{Y}}$ is a closed subgroup of $\mathbb{T}^{\mathbb{N}}$ which is weakly controllable but not controllable. Indeed, since the set $\langle Y\rangle$ is countable and $\mathbb{T}$ is uncountable, we can fix $x \in \mathbb{T} \backslash\langle Y\rangle$. By Claim $6, c_{x} \in \overline{D_{Y}}=H$, and Claim 5 can be applied.

Example 4.2. Let $\mathbb{P} \backslash\{2\}=\left\{p_{k}: k \in \mathbb{N}\right\}$ be a faithful enumeration of all prime numbers other than 2. For every $k \in \mathbb{N}$ let $y_{k} \in \mathbb{T}$ be one of the two elements of order $p_{k}$ which are closest to 0 (in the natural metric of $\mathbb{T}$ ). Then $Y=\left\{y_{k}: k \in \mathbb{N}\right\} \subseteq t(\mathbb{T})$ is a sequence converging to 0 in $\mathbb{T}$. Let $x \in \mathbb{T}$ be the element of order 2 . Then $H=\left\langle F_{Y} \cup\left\{c_{x}\right\}\right\rangle$ is a countable torsion subgroup of $\mathbb{T}^{\mathbb{N}}$ that is weakly controllable but not controllable. Clearly, $H$ is countable. Since $x$ has order 2 , the constant function $c_{x} \in \mathbb{T}^{\mathbb{N}}$ also has order 2 ; in particular, $c_{x} \in t\left(\mathbb{T}^{\mathbb{N}}\right)$. Combining this with (4.3), we conclude that $H=\left\langle F_{Y} \cup\left\{c_{x}\right\}\right\rangle \subseteq t\left(\mathbb{T}^{\mathbb{N}}\right)$; that is, $H$ is torsion. It remains only to show that $X=\{x\}$ and $H$ satisfy the assumption of Claim 7. Observe that every non-zero element of the group $\langle Y\rangle=\bigoplus_{k \in \mathbb{N}}\left\langle y_{k}\right\rangle$ has order $p_{1} p_{2} \ldots p_{i}$ for suitable $p_{1}, p_{2}, \ldots, p_{i} \in \mathbb{P} \backslash\{2\}$, so $\langle Y\rangle \backslash\{0\}$ has only elements of odd order. Since $x$ has an even order 2, we have $x \in \mathbb{T} \backslash\langle Y\rangle$. Thus, $x \in\{x\} \backslash\langle Y\rangle=X \backslash\langle Y\rangle \neq \emptyset$.

Theorem 4.3. Let $\mathscr{H}$ be the family of subgroups $H$ of $\mathbb{T}^{\mathbb{N}}$ which are weakly controllable but not controllable. Then $|\mathscr{H}|=2^{\mathfrak{c}}$.

Proof. Since $\left|\mathbb{T}^{\mathbb{N}}\right|=\mathfrak{c}$, we have $|\mathscr{H}| \leq 2^{\mathfrak{c}}$. It remains only to show that $|\mathscr{H}| \geq 2^{\mathfrak{c}}$.

Let $Y=\left\{y_{k}: k \in \mathbb{N}\right\} \subseteq t(\mathbb{T}) \backslash\{0\}$ be any sequence converging to 0 in $\mathbb{T}$. Since $\mathbb{T}$ has rank $\mathfrak{c}$, we can fix an independent set $Z \subseteq \mathbb{T} \backslash t(\mathbb{T})$ with $|Z|=\mathfrak{c}$. Then the family $\mathscr{X}=\{X \subseteq Z: X \neq \emptyset\}$ of all non-empty subsets of $Z$ has cardinality $2^{\mathfrak{c}}$. Since $Z$ is an independent subset of $\mathbb{T}$, the set $C=\left\{c_{x}: x \in Z\right\}$ is an independent subset of $\mathbb{T}^{\mathbb{N}}$.

For every $X \in \mathscr{X}$, define $H_{X}=\left\langle F_{Y} \cup\left\{c_{x}: x \in X\right\}\right\rangle$.

Claim 8. If $X_{0}, X_{1} \in \mathscr{X}$ and $X_{0} \neq X_{1}$, then $H_{X_{0}} \neq H_{X_{1}}$.

Proof. There exist $i \in\{0,1\}$ and $x^{*} \in X_{i} \backslash X_{1-i}$. Clearly, $c_{x^{*}} \in H_{X_{i}}$, so it remains only to show that $c_{x^{*}} \notin H_{X_{1-i}}$. Suppose that $c_{x^{*}} \in H_{X_{1-i}}=\left\langle F_{Y} \cup\left\{c_{x}: x \in X_{1-i}\right\}\right\rangle$. Then $c_{x^{*}}=g+h$ for some $g \in\left\langle F_{Y}\right\rangle$ and $h \in\left\langle\left\{c_{x}: x \in X_{1-i}\right\}\right\rangle$. Note that $X_{0} \cup X_{1} \subseteq Z$, so $g=c_{x^{*}}-h \in\langle C\rangle$. Since $g \in\left\langle F_{Y}\right\rangle$, we have $g \in t\left(\mathbb{T}^{\mathbb{N}}\right)$ by (4.3). Thus, $g \in\langle C\rangle \cap t\left(\mathbb{T}^{\mathbb{N}}\right)=\{0\}$ (as $C$ is independent). This gives $g=0$ and $c_{x^{*}}=h \in\left\langle\left\{c_{x}: x \in X_{1-i}\right\}\right\rangle$. Since $x^{*} \notin X_{1-i}, c_{x^{*}} \notin\left\{c_{x}: x \in X_{1-i}\right\}$. This contradicts the fact that $C$ is independent. 
Claim 9. For every $X \in \mathscr{X}$, the subgroup $H_{X}$ of $\mathbb{T}^{\mathbb{N}}$ is weakly controllable but not controllable.

Proof. Since $X \neq \emptyset$, we can fix $x \in X$. Since $X \subseteq Z$ and $Z$ is independent in $\mathbb{T}, x$ has infinite order in $\mathbb{T}$. Since $Y \subseteq t(\mathbb{T})$, we have $\langle Y\rangle \subseteq t(\mathbb{T})$. This yields $x \in X \backslash\langle Y\rangle$. Now the conclusion follows from Claim 7.

From Claims 8 and 9, we obtain the inequality $|\mathscr{H}| \geq\left|\left\{H_{X}: X \in \mathscr{X}\right\}\right|=|\mathscr{X}|=2^{\mathfrak{c}}$.

\section{Behavior of CONTROLlability UNDER TAKing CLOSURES AND DENSE SUbGRoups}

In this section we investigate how the three notions of controllability behave under the closure operation.

Proposition 5.1. Let $I$ be a set and $\left\{G_{i}: i \in I\right\}$ be a family of topological groups. Let $H$ and $H^{\prime}$ be subgroups of $G=\prod_{i \in I} G_{i}$ such that $H \subseteq H^{\prime} \subseteq \bar{H}$. If $H$ is weakly controllable in $G$, then so is $H^{\prime}$.

Corollary 5.2. The closure of a weakly controllable subgroup is weakly controllable.

Our next example shows that the situation changes completely in the case of controllability.

Example 5.3. The closure of a (countable torsion) controllable subgroup of $\mathbb{T}^{\mathbb{N}}$ need not be controllable. Indeed, let $H=\overline{D_{Y}}$ be the closed non-controllable subgroup of $\mathbb{T}^{\mathbb{N}}$ constructed in Example 4.1. Since $D_{Y} \subseteq \bigoplus_{n \in \mathbb{N}} t(\mathbb{T}) \subseteq \bigoplus_{n \in \mathbb{N}} \mathbb{T}$, it follows from Proposition 1.2(ii) that $D_{Y}$ is controllable.

Since the group $H$ in the above example is compact, this example shows that "uniformly" cannot be omitted either from our next theorem or from its Corollary 5.5 below.

Theorem 5.4. Let $I$ be a set and $\left\{G_{i}: i \in I\right\}$ be a family of topological groups. Furthermore, let $H$ be a subgroup of $G=\prod_{i \in I} G_{i}$ such that $\bar{H}$ is compact. If $H$ is uniformly controllable, then so is $\bar{H}$.

Proof. Let $J$ be a finite subset of $I$. Since $H$ is uniformly controllable, there exists a finite set $K \subseteq I$ such that

$$
p_{I J}(H)=p_{I J}\left(H \cap \bigoplus_{i \in K} G_{i}\right) \subseteq p_{I J}\left(\bar{H} \cap \bigoplus_{i \in K} G_{i}\right) .
$$

Since $K$ is finite, $\bigoplus_{i \in K} G_{i}$ is a closed subset of $G$, and so $\bar{H} \cap \bigoplus_{i \in K} G_{i}$ is a closed subset of $\bar{H}$. Since the latter set is compact, so is the former. As a continuous image of the compact set $\bar{H} \cap \bigoplus_{i \in K} G_{i}$, the last set in (5.1) is compact, and so it is closed in $\prod_{i \in J} G_{i}$. Combining this with the inclusion from (5.1), we get $\overline{p_{I J}(H)} \subseteq p_{I J}\left(\bar{H} \cap \bigoplus_{i \in K} G_{i}\right)$. Since the map $p_{I J}$ is continuous, we also have $p_{I J}(\bar{H}) \subseteq \overline{p_{I J}(H)}$. This yields $p_{I J}(\bar{H}) \subseteq p_{I J}\left(\bar{H} \cap \bigoplus_{i \in K} G_{i}\right)$. The inverse inclusion is obvious. This finishes the proof of uniform controllability of $\bar{H}$ in $G$.

Corollary 5.5. Let $\left\{G_{i}: i \in I\right\}$ be a family of compact groups and $H$ be a uniformly controllable subgroup of $G=\prod_{i \in I} G_{i}$. Then $\bar{H}$ is also uniformly controllable in $G$.

Remark 5.6. Let $D_{Y}$ be the controllable subgroup of $\mathbb{T}^{\mathbb{N}}$ mentioned in Example 5.3. Since its closure $H=\overline{D_{Y}}$ in $\mathbb{T}^{\mathbb{N}}$ is not controllable, it is not uniformly controllable either. Therefore, $D_{Y}$ is not uniformly controllable by Corollary 5.5.

Items (ii) and (iii) of our next example show that a dense subgroup of a uniformly controllable group need not be even weakly controllable, which shows that controllability is badly destroyed by passing to a dense subgroup. 
Example 5.7. Let $K$ be a non-trivial abelian topological group.

(i) There exists a dense subgroup $H$ of $K^{\mathbb{N}}$ such that $H \cap \bigoplus_{n \in \mathbb{N}} K=\{0\}$ and $|H| \leq \max \{w(K), \omega\}$, where $w(K)$ is the weight of $K$. Indeed, let $D$ be a dense subgroup of $K$ such that $|D|=w(K)$. Fix a partition $\mathbb{N}=\bigcup_{k \in \mathbb{N}} I_{k}$ of $\mathbb{N}$ into pairwise disjoint infinite sets $I_{k}$. For each $k \in \mathbb{N}$ define

$$
S_{k}=I_{k} \backslash\{0,1, \ldots, k\} .
$$

For $d \in D$ and $k \in \mathbb{N}$ we define $x_{d, k} \in K^{\mathbb{N}}$ by letting

$$
x_{d, k}(n)=\left\{\begin{array}{ll}
d, & \text { if } n \in S_{k} \cup\{k\} \\
0, & \text { if } n \in \mathbb{N} \backslash\left(S_{k} \cup\{k\}\right)
\end{array} \quad \text { for } n \in \mathbb{N} .\right.
$$

Since $D$ is a subgroup of $K$, it follows from (5.3) that $X_{k}=\left\{x_{d, k}: d \in D\right\}$ is a subgroup of $K^{\mathbb{N}}$ for every $k \in \mathbb{N}$. We claim that

$$
H=\left\langle\bigcup_{k \in \mathbb{N}} X_{k}\right\rangle
$$

is the desired subgroup of $K^{\mathbb{N}}$. Clearly, $\left|X_{k}\right|=|D|$ for every $k \in \mathbb{N}$, so $|H| \leq \max \{|D|, \omega\}=$ $\max \{w(K), \omega\}$.

Next, let us check that $H \cap \bigoplus_{n \in \mathbb{N}} K=\{0\}$. Choose $h \in H \backslash\{0\}$ arbitrarily. Since each $X_{k}$ is a subgroup of $K^{\mathbb{N}}$, it easily follows from (5.4) that $h=\sum_{i=0}^{j} x_{d_{i}, k_{i}}$ for suitable $d_{i}$ and $k_{i}$ $(i=0, \ldots, j)$ such that $k_{0}, k_{1}, \ldots, k_{j}$ are pairwise distinct. Since $h \neq 0$, there exists $l=0, \ldots, j$ such that $x_{d_{l}, k_{l}} \neq 0$. From this and (5.3) we conclude that $d_{l} \neq 0$. Since $S_{l}$ is infinite, the set $S=S_{l} \backslash\left\{k_{i}: i=0, \ldots, j\right\}$ is also infinite.

Let $n \in S$ be arbitrary. Since $S_{i} \cap S_{l}=\emptyset$ for all $i=0,1, \ldots, j$ with $i \neq l$, it follows from (5.3) and our definition of $S$ that $x_{d_{i}, k_{i}}(n)=0$ whenever $i=0,1, \ldots, j$ and $i \neq l$, so $h(n)=x_{d_{l}, k_{l}}(n)=$ $d_{l} \neq 0$. Since $S$ is infinite, this means that $h \notin \bigoplus_{n \in \mathbb{N}} K$. This finishes the proof of the equality $H \cap \bigoplus_{n \in \mathbb{N}} K=\{0\}$.

It remains only to show that $H$ is dense in $K^{\mathbb{N}}$. Fix an arbitrary non-empty open subset $W$ of $K^{\mathbb{N}}$. Then there exist $k \in \mathbb{N}$ and non-empty open subsets $U_{0}, \ldots, U_{k}$ of $K$ such that

$$
\left(\prod_{i=0}^{k} U_{i}\right) \times \prod_{j>k} K \subseteq W
$$

By (finite) induction on $j=0,1, \ldots, k$, we select $d_{j} \in D$ as follows. Since $D$ is dense in $K$, we fix $d_{0} \in D \cap U_{0}$. Using density of $D$ in $K$ again, we select $d_{1} \in D \cap\left(U_{1}-x_{d_{0}, 0}(1)\right)$. Assuming that $d_{0}, \ldots, d_{j-1}$ were already defined, we use density of $D$ in $K$ again to choose

$$
d_{j} \in D \cap\left(U_{j}-\sum_{i=0}^{j-1} x_{d_{i}, i}(j)\right)
$$

Note that

$$
h=\sum_{i=0}^{k} x_{d_{i}, i} \in H
$$

Fix $j \in\{0,1, \ldots, k\}$. If $i \in\{j+1, \ldots, k\}$, then $x_{d_{i}, i}(j)=0$ by (5.2) and (5.3). Combining this with (5.6) and (5.7), we obtain

$$
h(j)=\sum_{i=0}^{k} x_{d_{i}, i}(j)=\sum_{i=0}^{j} x_{d_{i}, i}(j)=\left(\sum_{i=0}^{j-1} x_{d_{i}, i}(j)\right)+x_{d_{j}, j}(j)=\left(\sum_{i=0}^{j-1} x_{d_{i}, i}(j)\right)+d_{j} \in U_{j} .
$$

Since (5.8) holds for every $j=0,1, \ldots, k$, from (5.5) we conclude that $h \in W$. From this and (5.7), we get $h \in W \cap H \neq \emptyset$. Therefore, $H$ is dense in $K^{\mathbb{N}}$. 
(ii) Let $H$ be the subgroup of $K^{\mathbb{N}}$ constructed in item (i). Clearly, $H$ is not weakly controllable, while $K^{\mathbb{N}}$ is uniformly controllable by Proposition 1.2(i). This shows that a dense subgroup of a uniformly controllable group need not be even weakly controllable.

(iii) If one takes $K$ to be finite, then the subgroup $H$ of $K^{\mathbb{N}}$ constructed in item (i) becomes countable. This allows us to conclude that a countable dense subgroup of a compact metric uniformly controllable group need not be even weakly controllable.

\section{Connections With CODing theory}

Let $\mathbb{Z}$ be the set of integer numbers. For $n \in \mathbb{Z}$, we let $n^{-}=\{k \in \mathbb{Z}: k \leq n\}$ and $n^{+}=\{k \in \mathbb{Z}$ : $k \geq n\}$.

Let us recall the classical notions from coding theory; see [13, 4, 3, 5].

Definition 6.1. Assume that $I=\mathbb{N}$ or $I=\mathbb{Z}$. Let $\left\{G_{i}: i \in I\right\}$ be a family of topological groups and $G=\prod_{i \in I} G_{i}$. A subgroup $H$ of $G$ is called:

(i) weakly controllable in $G$ provided that $H \cap \bigoplus_{i \in I} G_{i}$ is dense in $H$;

(ii) controllable in $G$ provided that for each pair $h, h^{\prime}$ of elements of $H$ and every integer $n \in I$ there exist an integer $m \in I$ and an element $g \in H$ such that $g \uparrow_{n^{-} \cap I}=h \uparrow_{n^{-} \cap I}$ and $g \uparrow_{m^{+} \cap I}=h^{\prime} \uparrow_{m^{+} \cap I}$

(iii) $k$-controllable in $G$, for a fixed $k \in \mathbb{N}$, provided that item (ii) holds with $m=n+k$;

(iv) strongly controllable in $G$ provided that $H$ is $k$-controllable for some $k \in \mathbb{N}$.

Note that the topology of $G_{i}$, if any, is only used in item (i) and is irrelevant in items (ii)-(iv).

These four notions play a prominent role in coding theory. According to Forney and Trott [5], a group code is a set of sequences that has a group property under a component-wise group operation. In this general setting, a group code may also be seen as the behavior of a behavioral group system as given by Willens $[14,15]$. It is known that many of the fundamental properties of linear codes and systems depend only on their group structure. In fact, Forney and Trott, loc. cit., obtain purely algebraic proofs of many of their results. These notions are used in the study of convolutional codes that are well known and used currently in data transmission (cf. [4]).

Definition 6.2. Assume that $I=\mathbb{N}$ or $I=\mathbb{Z}$. Let $\left\{G_{i}: i \in I\right\}$ be a family of groups and $G=\prod_{i \in I} G_{i}$. We say that a subgroup $H$ of $G$ is uniformly controllable in $G$ provided that for every integer $n \in I$ there exists an integer $m \in I$ such that for each pair $h, h^{\prime}$ of elements of $H$ one can find an element $g \in H$ satisfying $g \uparrow_{n^{-} \cap I}=h \uparrow_{n^{-} \cap I}$ and $g \uparrow_{m^{+} \cap I}=h^{\prime} \uparrow_{m^{+} \cap I}$.

One can easily see that

$$
\text { strongly controllable } \rightarrow \text { uniformly controllable } \rightarrow \text { controllable. }
$$

Clearly, our notion of weak controllability in Definition 1.4 is a direct generalization of the classical notion of weak controllability from Definition 6.1(i). The next proposition shows that our notion of controllability in Definition 1.1(i) is equivalent to the classical notion of controllability from Definition 6.1(ii) when $I=\mathbb{N}$.

Proposition 6.3. For every family $\left\{G_{i}: i \in \mathbb{N}\right\}$ of groups, a subgroup $H$ of $G=\prod_{i \in \mathbb{N}} G_{i}$ is controllable in $G$ in the sense of Definition 1.1(i) if and only if $H$ is controllable in $G$ in the sense of Definition 6.1(ii).

Proof. Assume that $H$ is controllable in $G$ in the sense of Definition 1.1(i). To check that $H$ is also controllable in $G$ in the sense of Definition 6.1(ii), fix $h, h^{\prime} \in H$ and $n \in \mathbb{N}$. Since $H$ is a subgroup of $G$, we have $h^{*}=h\left(h^{\prime}\right)^{-1} \in H$. Clearly, the set $J=n^{-} \cap \mathbb{N}$ is finite. By Proposition 1.3(i), there exists $g^{*} \in H \cap \bigoplus_{i \in \mathbb{N}} N$ such that $g^{*} \uparrow_{J}=h^{*} \uparrow_{J}$. Fix a finite set $K \subseteq \mathbb{N}$ with $g^{*} \in \bigoplus_{i \in K} G_{i}$. Since 
$L=J \cup K$ is finite, we can choose $m \in \mathbb{N}$ satisfying $m>\max L$. Since $g^{*}, h^{\prime} \in H$ and $H$ is a subgroup of $G$, one has $g=g^{*} h^{\prime} \in H$. If $i \in n^{-} \cap \mathbb{N}=J$, then

$$
g(i)=g^{*}(i) h^{\prime}(i)=h^{*}(i) h^{\prime}(i)=h(i)\left(h^{\prime}\right)^{-1}(i) h^{\prime}(i)=h(i) h^{\prime}(i)^{-1} h^{\prime}(i)=h(i),
$$

which yields $g \uparrow_{n^{-} \cap \mathbb{N}}=h \uparrow_{n^{-} \cap \mathbb{N}}$. If $i \in m^{+} \cap \mathbb{N}$, then $i \notin K$ by the choice of $m$, and so $g^{*}(i)=1$ by the choice of $K$; in particular, $g(i)=g^{*}(i) h^{\prime}(i)=h^{\prime}(i)$. This shows that $g \uparrow_{m^{+} \cap \mathbb{N}}=h^{\prime} \uparrow_{m^{+} \cap \mathbb{N}}$.

Suppose now that $H$ is controllable in $G$ in the sense of Definition 6.1(ii). To check that $H$ is controllable in $G$ in the sense of Definition 1.1(i), fix $h \in H$ and a finite set $J \subseteq \mathbb{N}$. Choose $n \in \mathbb{N}$ with $n \geq \max J$. Since $H$ is controllable in $G$ in the sense of Definition 6.1(ii), applying this definition to $h, h^{\prime}=1$ and $n$, we can find $m \in \mathbb{N}$ and $g \in H$ such that $g \uparrow_{n^{-} \cap \mathbb{N}}=h \uparrow_{n^{-} \cap \mathbb{N}}$ and $g \uparrow_{m^{+} \cap \mathbb{N}}=h^{\prime} \uparrow_{m^{+} \cap \mathbb{N}}=1$. Clearly, the set $K=m^{-} \cap \mathbb{N}$ is finite. From $g \uparrow_{m^{+} \cap \mathbb{N}}=1$ and our definition of $K$ we conclude that $g \in \bigoplus_{i \in K} G_{i} \subseteq \bigoplus_{i \in \mathbb{N}} G_{i}$. Since $J \subseteq n^{-} \cap \mathbb{N}$, we have $g(i)=g \uparrow_{n^{-} \cap I}(i)=h(i)$ for every $i \in J$. That is, $g \uparrow_{J}=h \uparrow_{J}$. Applying Proposition 1.3(i), we conclude that $H$ is controllable in $G$ in the sense of Definition 1.1(i).

Finally, it is worth pointing out that the notion of uniform controllability in Definition 1.1(ii) appears to be new even in the case when $I=\mathbb{N}$. The next proposition shows that in this special case it coincides with the notion from Definition 6.2.

Proposition 6.4. For every family $\left\{G_{i}: i \in \mathbb{N}\right\}$ of groups, a subgroup $H$ of $G=\prod_{i \in \mathbb{N}} G_{i}$ is uniformly controllable in $G$ in the sense of Definition 1.1(ii) if and only if $H$ is uniformly controllable in $G$ in the sense of Definition 6.2.

The proof of this proposition is similar to that of Proposition 6.3, so we omit it.

The main advantage of our Definition 1.1 over the classical Definition 6.1, as well as the adapted Definition 6.2 , is that the index set $I$ is no longer required to be a subset of $\mathbb{Z}$; in particular, the order structure on $I$ becomes irrelevant.

It is worth emphasizing, based on Propositions 6.3 and 6.4, that all our results in the first five sections are applicable to the classical case $I=\mathbb{N}$. Furthermore, many of these results are new even in this classical case.

A straightforward proof of the following proposition is left to the reader.

Proposition 6.5. Let $M$ be a topological group and $H$ be a subgroup of $G=M^{\mathbb{N}}$. Then $H^{\prime}=$ $M^{\mathbb{Z} \backslash \mathbb{N}} \times H$ is a subgroup of $M^{\mathbb{Z} \backslash \mathbb{N}} \times M^{\mathbb{N}}=M^{\mathbb{Z}}=G^{\prime}$ satisfying the following conditions:

(i) $H$ is weakly controllable in $G$ in the sense of Definition 1.4 if and only if $H^{\prime}$ is weakly controllable in $G^{\prime}$ in the sense of Definition 6.1(i).

(ii) $H$ is controllable in $G$ in the sense of Definition 1.1(i) if and only if $H^{\prime}$ is controllable in $G^{\prime}$ in the sense of Definition 6.1(ii).

(iii) $H$ is uniformly controllable in $G$ in the sense of Definition 1.1(ii) if and only if $H^{\prime}$ is uniformly controllable in $G^{\prime}$ in the sense of Definition 6.2.

(iv) $H$ is closed in $G$ if and only if $H^{\prime}$ is closed in $G^{\prime}$.

This proposition allows us to "transform" our examples distinguishing properties in Definitions 1.1 and 1.4 into examples distinguishing corresponding properties from the classical Definition 6.1 and related Definition 6.2.

Combining Theorem 4.3 with items (i) and (ii) of Proposition 6.5, we obtain the following corollary.

Corollary 6.6. Let $\mathscr{H}$ be the family of subgroups $H$ of $G=\mathbb{T}^{\mathbb{Z}}$ which are weakly controllable but not controllable in $G$ in the sense of Definition 6.1 (in which we let $I=\mathbb{Z}$ ). Then $|\mathscr{H}|=2^{\mathfrak{c}}$.

Combining Example 5.3(i) with items (i), (ii) and (iv) of Proposition 6.5, we get the following corollary. 
Corollary 6.7. There exists a closed (and thus, compact) subgroup $H$ of $G=\mathbb{T}^{\mathbb{Z}}$ which is weakly controllable but not controllable in $G$ in the sense of Definition 6.1 (in which we let $I=\mathbb{Z}$ ).

Combining Example 5.6 with items (ii) and (iii) of Proposition 6.5, we obtain the following corollary.

Corollary 6.8. There exists a subgroup $H$ of $G=\mathbb{T}^{\mathbb{Z}}$ which is controllable in $G$ in the sense of Definition 6.1 but is not uniformly controllable in $G$ in the sense of Definition 6.2; in particular, $H$ is not strongly controllable in $G$ in the sense of Definition 6.1. (Here we take $I=\mathbb{Z}$ in all definitions.)

\section{UNIFORM CONTROLLABILITY VS STRONG CONTROLLABILITY}

Our next theorem provides a general technique for building uniformly controllable subgroups that are not strongly controllable.

Theorem 7.1. Let $I=\mathbb{N}$ or $I=\mathbb{Z}$. Let $\left\{G_{i}: i \in I\right\}$ be a family of topological groups and let $G=\prod_{i \in I} G_{i}$ be its direct product with the Tychonoff product topology. Suppose that $\left\{x_{j}: j \in \mathbb{N}\right\} \subseteq$ $\left(\bigoplus_{i \in I} G_{i}\right) \backslash\{0\}$ and $H$ is a subgroup of $G$ satisfying two conditions:

(i) $\left\{x_{j}: j \in \mathbb{N}\right\} \subseteq H \subseteq \overline{\left\langle\left\{x_{j}: j \in \mathbb{N}\right\}\right\rangle}$;

(ii) $\max \left(\operatorname{supp}\left(x_{s}\right)\right)<\min \left(\operatorname{supp}\left(x_{t}\right)\right)$ whenever $s, t \in \mathbb{N}$ and $s<t$.

Then $H$ is uniformly controllable in $G$.

Furthermore, assume also that the following conditions are satisfied:

(iii) $\lim _{j \rightarrow \infty}\left|\operatorname{supp}\left(x_{j}\right)\right|=\infty$;

(iv) if $j \in \mathbb{N}$ and $l, m \in \operatorname{supp}\left(x_{j}\right)$, then $x_{j}(l)$ and $x_{j}(m)$ have the same order.

Then $H$ is not strongly controllable in $G$.

Proof. Let $K=\overline{\left\langle\left\{x_{j}: j \in \mathbb{N}\right\}\right\rangle}$. It follows from (ii) that $\operatorname{supp}\left(x_{i}\right) \cap \operatorname{supp}\left(x_{j}\right)=\emptyset$ for $i, j \in \mathbb{N}$ with $i \neq j$. This easily implies that $\left\langle\left\{x_{j}: j \in \mathbb{N}\right\}\right\rangle=\bigoplus_{j \in \mathbb{N}}\left\langle x_{j}\right\rangle$ and $K \cong \prod_{j \in \mathbb{N}}\left\langle x_{j}\right\rangle$. Since $H \subseteq K$ by (i), for every $h \in H$ there exists a sequence $\left\{s_{j}: j \in \mathbb{N}\right\}$ of integer numbers so that

$$
h=\sum_{j=0}^{\infty} s_{j} x_{j} .
$$

Claim 10. $H$ is uniformly controllable in $G$.

Proof. Fix an integer $n \in I$. Apply (ii) to find $l \in \mathbb{N}$ such that

$$
n \leq \max \left(\operatorname{supp}\left(x_{l}\right)\right) \text {. }
$$

We claim that the integer

$$
m=\min \left(\operatorname{supp}\left(x_{l+1}\right)\right)
$$

satisfies Definition 6.2. Clearly, $m \in I$.

Let $h, h^{\prime} \in H$. Let (7.1) be the representation of $h$ and let

$$
h^{\prime}=\sum_{j=0}^{\infty} s_{j}^{\prime} x_{j}
$$

be a similar representation of $h^{\prime}$, for a suitable sequence $\left\{s_{j}^{\prime}: j \in \mathbb{N}\right\}$ of integer numbers.

Since $x_{0}, \ldots, x_{l} \in H$ and $H$ is a subgroup of $G$, we have

$$
y=\sum_{j=0}^{l} s_{j} x_{j} \in H \quad \text { and } \quad y^{\prime}=\sum_{j=0}^{l} s_{j}^{\prime} x_{j} \in H .
$$


Since $h^{\prime}=\sum_{j=0}^{\infty} s_{j}^{\prime} x_{j} \in H$ and $H$ is a subgroup of $G$, it follows that

$$
z=\sum_{j=l+1}^{\infty} s_{j}^{\prime} x_{j}=h^{\prime}-y^{\prime} \in H .
$$

From $y \in H$ and $z \in H$, we conclude that $g=y+z \in H$.

Let $i \in I$ and $i \leq n$. From (ii) and (7.2), it follows that $x_{j}(i)=0$ for all integers $j \geq l+1$, which yields

$$
h(i)=\sum_{j=0}^{\infty} s_{j} x_{j}(i)=\sum_{j=0}^{l} s_{j} x_{j}(i)=y(i)
$$

by (7.1) and (7.5). Furthermore, from (7.6) we get $z(i)=0$. Therefore, $g(i)=y(i)+z(i)=y(i)=$ $h(i)$. This shows that $g \uparrow_{n^{-} \cap I}=h \uparrow_{n^{-} \cap I}$.

Suppose now that $i \in I$ and $i \geq m$. From (ii) and (7.3), it follows that $x_{j}(i)=0$ for all integers $j=0, \ldots, l$, which yields

$$
h^{\prime}(i)=\sum_{j=0}^{\infty} s_{j}^{\prime} x_{j}(i)=\sum_{j=l+1}^{\infty} s_{j}^{\prime} x_{j}(i)=z(i)
$$

by (7.4) and (7.6). Furthermore, from (7.5) we get $y(i)=0$. Therefore, $g(i)=y(i)+z(i)=z(i)=$ $h^{\prime}(i)$. This shows that $g \uparrow_{m^{+} \cap I}=h^{\prime} \uparrow_{m^{+} \cap I}$.

Suppose now that conditions (iii) and (iv) hold. We are going to show that, under these additional assumptions, the group $H$ is not strongly controllable in $G$.

Indeed, suppose the contrary. Then $H$ is $k$-controllable in $G$ for some $k \in \mathbb{N}$; see Definition 6.1. Use (iii) to fix $l \in \mathbb{N}$ such that $\left|\operatorname{supp}\left(x_{l}\right)\right|>k+1$. Let $n=\min \operatorname{supp}\left(x_{l}\right)$ and $m=n+k$. Clearly, $n \in I$. Note that $x_{l} \in H$ by (i). Since $0 \in H$ and $H$ is $k$-controllable in $G$, there exists $g \in H$ such that $g \uparrow_{n^{-} \cap I}=x_{l} \uparrow_{n^{-} \cap I}$ and $g \uparrow_{m^{+} \cap I}=0$. In particular, $g(n)=x_{l}(n) \neq 0$, as $n \in \operatorname{supp}\left(x_{l}\right)$.

Since $g \in H$, there exists a sequence of integers $\left\{s_{j}: j \in \mathbb{N}\right\}$ such that $g=\sum_{j=0}^{\infty} s_{j} x_{j}$. From $g \uparrow_{n^{-} \cap I}=x_{l} \uparrow_{n^{-} \cap I}$ and (ii) we conclude that $s_{j} x_{j}=0$ for all $j<l$. Similarly, from $g \uparrow_{m^{+} \cap I}=0$ and (ii) we conclude that $s_{j} x_{j}=0$ for all $j>l$. This shows that $g=s_{l} x_{l}$.

Since $n=\min \operatorname{supp}\left(x_{l}\right)$ and $\left|\operatorname{supp}\left(x_{l}\right)\right|>k+1$, we conclude that $m=n+k<\max \operatorname{supp}\left(x_{l}\right)=i$. Therefore, $i \in m^{+} \cap I$, and so $s_{l} x_{l}(i)=g(i)=0$. Since $i, n \in \operatorname{supp}\left(x_{l}\right)$ and $s_{l} x_{l}(n)=g(n) \neq 0$, this contradicts (iv).

Example 7.2. Let $p$ be a prime number and let $G=\mathbb{Z}(p)^{\mathbb{Z}}$. Choose any sequence $\left\{x_{j}: j \in \mathbb{N}\right\} \subseteq$ $\left(\bigoplus_{n \in \mathbb{Z}} \mathbb{Z}(p)\right) \backslash\{0\}$ satisfying conditions (ii) and (iii) of Theorem 7.1, in which we take $I=\mathbb{Z}$ and $G_{i}=\mathbb{Z}(p)$ for every $i \in \mathbb{Z}$. Clearly, this sequence automatically satisfies the condition (iv) of this theorem as well.

(i) $K=\overline{\left\langle\left\{x_{j}: j \in \mathbb{N}\right\}\right\rangle}$ is a closed subgroup of $G$ which is uniformly controllable in $G$ but not strongly controllable in $G$. This follows from Theorem 7.1, as $H=K$ satisfies the condition (i) of this theorem.

(ii) $H=\left\langle\left\{x_{j}: j \in \mathbb{N}\right\}\right\rangle$ is a countable subgroup of $G$ which is uniformly controllable in $G$ but not strongly controllable in $G$. This also follows from Theorem 7.1, as $H$ satisfies the condition (i) of this theorem.

Theorem 7.3. Let $p$ be a prime number and let $\mathscr{H}_{p}$ be the family of subgroups of the product $G=\mathbb{Z}(p)^{\mathbb{N}}$ which are uniformly controllable in $G$ but not strongly controllable in $G$. Then $\left|\mathscr{H}_{p}\right|=2^{\mathfrak{c}}$.

Proof. Choose any sequence $\left\{x_{j}: j \in \mathbb{N}\right\} \subseteq\left(\bigoplus_{n \in \mathbb{Z}} \mathbb{Z}(p)\right) \backslash\{0\}$ satisfying the conditions (ii) and (iii) of Theorem 7.1. Clearly, this sequence automatically satisfies the condition (iv) of this theorem as well. Note that $K=\overline{\left\langle\left\{x_{j}: j \in \mathbb{N}\right\}\right\rangle}$ is topologically isomorphic to $\prod_{j \in \mathbb{N}}\left\langle\left\{x_{j}: j \in \mathbb{N}\right\}\right\rangle \cong \mathbb{Z}(p)^{\mathbb{N}}$, 
so $|K|=\left|\mathbb{Z}(p)^{\mathbb{N}}\right|=\mathfrak{c}$. Therefore, we can fix a set $X \subseteq K$ such that $|X|=\mathfrak{c}$ and $\langle X\rangle \cap\left\langle\left\{x_{j}: j \in\right.\right.$ $\mathbb{N}\}\rangle=\{0\}$. Then the set $\mathscr{Y}$ of all subsets of $X$ has cardinality $2^{\mathfrak{c}}$.

For every $Y \in \mathscr{Y}$, let $H_{Y}=\left\langle\left\{x_{j}: j \in \mathbb{N}\right\} \cup Y\right\rangle$ and note that $H_{Y}$ satisfies the condition (i) of Theorem 7.1. Applying Theorem 7.1, we conclude that each $H_{Y}$ is uniformly controllable but not strongly controllable in $G$, so $H_{Y} \in \mathscr{H}_{p}$. Since $H_{Y} \neq H_{Y^{\prime}}$ whenever $Y, Y^{\prime} \in \mathscr{Y}$ and $Y \neq Y^{\prime}$, it follows that $\left|\mathscr{H}_{p}\right| \geq\left|\left\{H_{Y}: Y \in \mathscr{Y}\right\}\right| \geq|\mathscr{Y}|=2^{\mathfrak{c}}$. Since $|K|=\mathfrak{c}$ and $H \subseteq K$ for every $H \in \mathscr{H}_{p}$, the reverse inequality $\left|\mathscr{H}_{p}\right| \leq 2^{\mathfrak{c}}$ holds as well.

\section{Compact products of COUntably many CyClic Groups}

In this section we use the Pontryagin duality to obtain characterizations of compact products of countably many cyclic groups.

Let $G$ be an arbitrary topological abelian group. A character on $G$ is a continuous homomorphism from $G$ to the circle group $\mathbb{T}$. The pointwise sum of two characters is again a character, and the set $\widehat{G}$ of all characters on $G$ is a group with pointwise addition as the composition law. If $G$ is locally compact, then the group $\widehat{G}$ equipped with the compact open topology becomes a topological group called the Pontryagin dual group of $G$. Many topological properties of a compact abelian group $G$ can be described via algebraic properties of the discrete dual $\widehat{G}$ of $G$; see $[2,7]$. For example, we have the following relations:

- $w(G)=|\widehat{G}|$

- $G$ is connected if and only if $\widehat{G}$ is torsion-free;

- $G$ is profinite if and only if $\widehat{G}$ is torsion.

For a fixed prime number $p$, an abelian group $A$ is called a $p$-group if $A=\left\{x \in A: p^{n} x=0\right.$ for some $n \in \mathbb{N}\}$.

Lemma 8.1. Let $p \in \mathbb{P}$ and let $A$ be an abelian p-group. If the torsion part $t(\widehat{A})$ of $\widehat{A}$ is dense in $\widehat{A}$, then $\bigcap_{n \in \mathbb{N}} p^{n} A=\{0\}$.

Proof. Suppose that $a \in \bigcap_{n \in \mathbb{N}} p^{n} A$ and $a \neq 0$. Since $A$ is $q$-divisible for every $q \in \mathbb{P} \backslash\{p\}$, it follows that $a \in \bigcap_{n \in \mathbb{N}^{+}} n A$. For every $n \in \mathbb{N}$ fix $a_{n} \in A$ such that $n a_{n}=a$. Since $a \neq 0$ and $A$ is discrete, there exists $\chi_{0} \in \widehat{A}$ such that $\chi_{0}(a) \neq 0$. Therefore, $U=\{\chi \in \widehat{A}: \chi(a) \neq 0\}$ is a non-empty open subset of $\widehat{A}$. By our assumption, there exists $\chi \in t(\widehat{A}) \cap U$. Therefore, $n \chi=0$ for some $n \in \mathbb{N}^{+}$. On the other hand, since $\chi \in U$ is a homomorphism, $n \chi\left(a_{n}\right)=\chi\left(n a_{n}\right)=\chi(a) \neq 0$, which shows that $n \chi \neq 0$, a contradiction.

Theorem 8.2. If $G$ is a compact metric profinite abelian group such that $t(G)$ is dense in $G$, then $G$ is topologically isomorphic to a product of countably many cyclic groups.

Proof. Since $G$ is compact metric abelian group, $A=\widehat{G}$ is a countable discrete group. Since $G$ is profinite, $A$ is a torsion group. Therefore, $A=\bigoplus_{p \in \mathbb{P}} A_{p}$, where each $A_{p}$ is a $p$-group.

Fix $p \in \mathbb{P}$. Since $A_{p}$ is a direct summand of $A$, there exists a continuous surjective homomorphism $f_{p}: G=\widehat{A} \rightarrow \widehat{A_{p}}$. Since $t(G)$ is dense in $G$ and $f_{p}$ is continuous, $f_{p}(t(G))$ must be dense in $\widehat{A_{p}}$. Since $f_{p}(t(G)) \subseteq t\left(\widehat{A_{p}}\right)$, we conclude that $t\left(\widehat{A_{p}}\right)$ is also dense in $\widehat{A_{p}}$. Since $A_{p}$ is a $p$-group, from Lemma 8.1 it follows that $\bigcap_{n \in \mathbb{N}} p^{n} A_{p}=\{0\}$; that is, $A_{p}$ does not have non-zero elements of infinite height. Since $A_{p}$ is countable, by the second Prüfer theorem, $A_{p}$ is a direct sum of (countably many) cyclic groups; see [6].

Since $A_{p}$ is a direct sum of cyclic groups for every $p \in \mathbb{P}$, so is $A=\bigoplus_{p \in \mathbb{P}} A_{p}$. By taking the dual, we conclude that $G=\widehat{A}$ is the direct product of countably many cyclic groups.

Corollary 8.3. For an abelian group $G$, the following conditions are equivalent:

(i) $G$ is topologically isomorphic to a direct product of countably many finite cyclic groups; 
(ii) $G$ is topologically isomorphic to a direct product of countably many finite groups;

(iii) $G$ is zero-dimensional, compact metric and $t(G)$ is dense in $G$.

Proof. The implication (i) $\rightarrow$ (ii) is trivial.

(ii) $\rightarrow$ (iii) Suppose that $G \cong \prod_{n \in \mathbb{N}} F_{n}$, where $F_{n}$ is a finite group. Then $G$ is clearly compact, zero-dimensional and metric. Furthermore, $D=\bigoplus_{n \in \mathbb{N}} F_{n}$ is dense in $G$. Since $t(G)$ contains $D$, it follows that $t(G)$ is also dense in $G$.

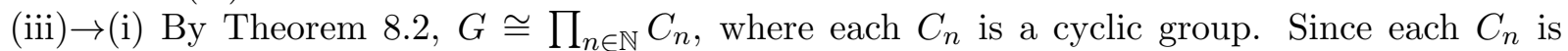
compact, as a continuous image of the compact space $G$, it must be finite.

Corollary 8.4. For a compact metric abelian group $G$, the following conditions are equivalent:

(i) $G$ is topologically isomorphic to a direct product of cyclic groups;

(ii) $G$ is profinite and $t(G)$ is dense in $G$.

\section{IN CONCLUSION}

Numke studied in [10] closed subgroups of the powers $\mathbb{Z}^{I}$ of the integers $\mathbb{Z}$ with the discrete topology. For a countable set $I$, he showed that:

- every closed subgroup of $\mathbb{Z}^{I}$ is itself a (direct) product,

- every endomorphism of $\mathbb{Z}^{I}$ is continuous, and

- every direct summand of $\mathbb{Z}^{I}$ is closed (and therefore, is itself a product).

Our next corollary can be considered as an analogue of this theorem for countable products of finite cyclic groups.

Corollary 9.1. Let $I$ be a countable set, $\left\{G_{i}: i \in I\right\}$ be a family of finite abelian groups and $G=\prod_{i \in I} G_{i}$ be its direct product. Then every closed weakly controllable subgroup $H$ of $G$ is topologically isomorphic to a direct product of finite cyclic groups.

Proof. Since all groups $G_{i}$ are finite, $\bigoplus_{i \in I} G_{i} \subseteq t(G)$, and so $H \cap\left(\bigoplus_{i \in I} G_{i}\right) \subseteq H \cap t(G)=t(H)$. Since $H$ is weakly controllable in $G, H \cap\left(\bigoplus_{i \in I} G_{i}\right)$ is dense in $H$. It follows that $t(H)$ is also dense in $H$. Clearly, $G$ is a compact profinite metric group, and so is its closed subgroup $H$. Applying the implication (ii) $\rightarrow$ (i) of Corollary 8.4 to $H$, we conclude that $H$ is topologically isomorphic to a direct product $\prod_{j \in J} C_{j}$ of cyclic groups $C_{j}$. Since each $C_{j}$ is a closed subgroup of the compact group $H$, it is also compact. Therefore, each $C_{j}$ must be finite.

Since direct products of finite groups are compact, the closedness of $H$ in $G$ is a necessary condition for $H$ to be topologically isomorphic to a direct product of finite groups. The next example shows that Corollary 9.1 fails without the assumption that $H$ is weakly controllable in $G$.

Example 9.2. Let $p$ be a prime number. For every $i \in \mathbb{N}$ let $G_{i}=\mathbb{Z}\left(p^{i}\right)$ be the cyclic group of order $p^{i}$. Then the direct product $G=\prod_{i \in \mathbb{N}} G_{i}$ contains a closed subgroup $H$ topologically isomorphic to the group $\mathbb{Z}_{p}$ of $p$-adic integers. (Indeed, use the fact that $\mathbb{Z}_{p}$ is topologically isomorphic to the limit of the inverse sequence of $G_{i}=\mathbb{Z}\left(p^{i}\right)$; see [2].) On the other hand, $\mathbb{Z}_{p}$ is known to be indecompasable; that is, $\mathbb{Z}_{p}$ cannot be represented as a product of two topological groups; see [2].

Recall that a compact group $G$ is a Valdivia compact group provided that $G$ is homeomorphic to the subspace

$$
\left\{x \in[0,1]^{I}:\{i \in I: x(i) \neq 0\} \text { is countable }\right\}
$$

of the Tychonoff cube $[0,1]^{I}$ for some index set $I$; see $[9]$. Several important results have been proved for these groups recently $[8,1,9]$. Chigogidze proves in [1] that every Valdivia compact group is homeomorphic to a product of metrizable compacta. Kubis [8] considers the smallest class $\mathcal{R}$ of compact spaces containing all compact metric spaces and closed under limits of continuous inverse sequences of retractions, and he shows that every compact connected abelian group which 
is a topological retract of a space from class $\mathcal{R}$ is homeomorphic to a product of compact metric spaces.

Our Corollary 9.1 could be compared with the above results. It seems worth noting that in Corollary 9.1 one has a topological isomorphism, while results mentioned above give only a homeomorphism (discarding the algebraic structure).

Remark 9.3. Apparently, Staiger [12] was the first to suggest that taking closed subgroups of direct products is crucial for obtaining good properties of group codes they represent. Most (although not all) of the examples in our paper deal with non-closed subgroups of direct products. This provides a strong evidence in support of the validity of Staiger's hypothesis.

For convenience of specialists in coding theory, we restate here some of our results in terms commonly used in coding theory.

Corollary 9.4. Let $\mathcal{C}$ be a complete group code in $G=\prod_{i \in \mathbb{N}} G_{i}$, where every group $G_{i}$ is finite. Then the following conditions are equivalent:

(1) $\mathcal{C}$ is weakly controllable;

(2) $\mathcal{C}$ is controllable;

(3) $\mathcal{C}$ is uniformly controllable.

Furthermore, if all $G_{i}$ are abelian, then $\mathcal{C}$ is topologically isomorphic to a direct product of finite cyclic groups.

Proof. A "complete group code in $G$ " means "closed subgroup of $G$ ", so $\mathcal{C}$ is a closed subgroup of $G$. Therefore, the equivalence of (1), (2) and (3) follows from Corollary 2.3. The final statement is proved in Corollary 9.1.

Remark 9.5. When the code $\mathcal{C}$ in Corollary 9.4 is in addition time invariant, then it follows from condition (3) that all properties in this corollary are equivalent to the strong controllability of $\mathcal{C}$; see [3].

\section{REFERENCES}

[1] A. Chigogidze, Valdivia compact groups are products, Top. and Appl., vol 155, pp. 605-609, 2008.

[2] D. Dikranjan, Iv. Prodanov and L. Stoyanov, Topological Groups: Characters, Dualities and Minimal Group Topologies, Pure and Applied Mathematics, vol. 130, Marcel Dekker Inc., New York-Basel (1989).

[3] F. Fagnani, Shifts on compact and discrete Lie groups: Algebraic topological invariants and classification problems, Adv. Math., vol. 127, pp. 283-306, 1997.

[4] G. D. Forney, Jr. and M. D. Trott, The dynamics of group codes: State spaces, trellis diagrams and canonical encoders, IEEE Trans. Inform. Theory, vol. 39, pp. 1491-1513, Sept. 1993.

[5] G. D. Forney, Jr. and M. D. Trott, The Dynamics of Group Codes: Dual Abelian Group Codes and Systems, Proc. IEEE Workshop on Coding, System Theory and Symbolic Dynamics (Mansfield, MA), pp. 35-65 (2004).

[6] L. Fuchs, Infinite Abelian Groups, Vol. I and II, Academic Press, 1970 and 1973.

[7] K.-H. Hofmann and S.A. Morris, The structure of compact groups. A primer for the student - a handbook for the expert, Second revised and augmented edition (de Gruyter Studies in Mathematics, 25), Walter de Gruyter \& Co., Berlin, 2006.

[8] W. Kubiś, Valdivia compact abelian groups, Rev. R. Acad. Cienc. Exactas Fís. Nat. Ser. A Math. RACSAM, vol. 102, pp. 193-197, 2008.

[9] W. Kubiś, and V. Uspenskij, A compact group which is not Valdivia compact, Proc. Amer. Math. Soc., vol. 133, pp. 2483-2487, 2005.

[10] R.J. Nunke, On direct products of infinite cyclic groups, Proc. Amer. Math. Soc., vol. 13, pp. 66-71, 1962.

[11] J. Rosenthal, S.M. Schumacher and E.V. York, On Behaviors and Convolutional Codes, IEEE Trans. on Information Theory, 42 (1996), no. 6, 1881-1891.

[12] L. Staiger, Convolutional codes as subgroups of $G F(q)^{\omega}$, Problemy Peredachi Informatsii, vol. 16, no. 4, pp. 98-101, (1980). (Russian).

[13] M. D. Trott, The algebraic structure of trellis codes, Ph. D. dissertation, Stanford Univ., Dept. Elec. Eng., Standford, CA, 1992. 
[14] J. C. Willems, From time series to linear systems, Parts I-III, vol. 22, pp. 561-580 and 675-694, 1986.

[15] J. C. Willems, On interconnections, control and feedback, IEEE Trans. Automat. Contr., vol. 42, pp. 326-339, Mar. 1997.

(M. Ferrer) Universitat Jaume I, Instituto de Matemáticas de Castellón, Campus de Riu Sec, 12071 CAstellón, SPAin.

E-mail address: mferrer@mat.uji.es

(S. Hernández) Universitat Jaume I, Departamento de Matemáticas, Campus de Riu Sec, 12071 Castellón, Spain.

E-mail address: hernande@mat.uji.es

(D. Shakhmatov) Division of Mathematics, Physics and Earth Sciences, Graduate School of Science And Engineering, Ehime University, Matsuyama 790-8577, JaPAN

E-mail address: dmitri.shakhmatov@ehime-u.ac.jp 